\title{
The algebraic surfaces on which the classical Phragmén-Lindelöf theorem holds
}

Received: 15 March 2005 / Published online: 26 January 2006

(C) Springer-Verlag 2006

\begin{abstract}
Let $V$ be an algebraic variety in $\mathbb{C}^{n}$. We say that $V$ satisfies the strong Phragmén-Lindelöf property (SPL) or that the classical Phragmén-Lindelöf Theorem holds on $V$ if the following is true: There exists a positive constant $A$ such that each plurisubharmonic function $u$ on $V$ which is bounded above by $|z|+o(|z|)$ on $V$ and by 0 on the real points in $V$ already is bounded by $A|\operatorname{Im} z|$. For algebraic varieties $V$ of pure dimension $k$ we derive necessary conditions on $V$ to satisfy (SPL) and we characterize the curves and surfaces in $\mathbb{C}^{n}$ which satisfy (SPL). Several examples illustrate how these results can be applied.
\end{abstract}

Mathematics Subject Classification (2000) 31C10 - 32C25 · 32U05

\section{Introduction}

An algebraic variety $V$ in $\mathbb{C}^{n}, n \geq 2$, has the property (SPL) if there exists a constant $A \geq 1$ such that for each plurisubharmonic function $u$ on $V$ the estimates

$$
u(z) \leq|z|+o(|z|), z \in V, \text { and } u(z) \leq 0, z \in V \cap \mathbb{R}^{n},
$$

imply

$$
u(z) \leq A|\operatorname{Im} z|, \quad z \in V
$$

R.W. Braun

Mathematisches Institut, Heinrich-Heine-Universität, 40225 Düsseldorf, Germany

E-mail: Ruediger.Braun@uni-duesseldorf.de

R. Meise ( $\varangle)$

Mathematisches Institut, Heinrich-Heine-Universität, 40225 Düsseldorf, Germany

E-mail: meise@math.uni-duesseldorf.de

B.A. Taylor

Department of Mathematics, University of Michigan, Ann Arbor, MI 48109, USA

E-mail: taylor@umich.edu 
By the classical Phragmén-Lindelöf Theorem, $V=\mathbb{C}^{n}$ satisfies (SPL) with $A=1$. Thus the varietes $V$ that have the property (SPL) are the ones for which a natural extension of the classical Phragmén-Lindelöf Theorem holds. This, however, is not the only reason why they are of interest. In fact, there are several problems concerning linear partial differential operators with constant coefficients for which the property (SPL) plays an important role in their solution. The first result of this type was obtained in Meise and Taylor [14]. There it was shown that for a homogeneous polynomial $P_{m} \in \mathbb{C}\left[z_{1}, \ldots, z_{n}\right]$ of degree $m \geq 2$ and $P\left(z^{\prime}, z_{n+1}\right):=P_{m}\left(z^{\prime}\right)-z_{n+1}$, the operator $P(D): C^{\infty}\left(\mathbb{R}^{n+1}\right) \rightarrow C^{\infty}\left(\mathbb{R}^{n+1}\right)$ admits a continuous linear right inverse if and only if the variety $V(P):=\left\{z \in \mathbb{C}^{n+1}: P(-z)=0\right\}$ satisfies (SPL). For $n=3$ the latter property was characterized in [3] by properties of $P_{m}$ (for the precise formulation, see Theorem 5.10). For a weight function $\omega$ (e.g., $\left.\omega(t)=t^{\alpha}, 0<\alpha<1\right)$ and $P \in \mathbb{C}\left[z_{1}, \ldots, z_{n}\right]$ we showed in [7] that the operator $P(D)$, acting on the space of $\omega$-ultradifferentiable functions $\mathcal{E}_{\omega}\left(\mathbb{R}^{n}\right)$ of Beurling type, admits a continuous linear right inverse only if certain limit varietes $T_{\gamma, d} V(P)$ associated with $V(P)$ have the property (SPL). A modification of this result by Heinrich [11] together with a theorem of Hörmander [12] shows that $P(D)$ acts surjectively on the space $\mathcal{A}\left(\mathbb{R}^{n}\right)$ of all real analytic functions on $\mathbb{R}^{n}$ only if for each $\xi \in V\left(P_{m}\right) \cap \mathbb{R}^{n},|\xi|=1$, all limit varieties $T_{\sigma, \delta}\left(V\left(P_{m}\right)-\xi\right)$ satisfy (SPL), where $P_{m}$ is the principal part of $P$ and where the limit varieties are computed at the singular point $0 \in\left(V\left(P_{m}\right)-\xi\right)$.

In the present paper we characterize the algebraic curves and surfaces $V$ in $\mathbb{C}^{n}$ which satisfy (SPL) in terms of their geometry. To achieve this, we first collect necessary conditions in Section 3 for any algebraic variety $V$ in $\mathbb{C}^{n}$ of pure dimention $k$ to satisfy (SPL). The first one was noted already in Meise and Taylor [14], namely that at $\xi \in V \cap \mathbb{R}^{n}$, the variety $V$ must satisfy the local Phragmén-Lindelöf condition $\mathrm{PL}_{\mathrm{loc}}(\xi)$ that was introduced in Hörmander [12]. The second and the third one were proved in [7]. They are formulated in terms of limit varieties $T_{\gamma, d} V$ of order $d \geq 1$ along real simple curves $\gamma$, which we briefly introduce in Section 2 . They state that each limit variety $T_{\gamma, d} V$ satisfies (SPL) if $V$ satisfies (SPL) and that for each real regular point $\xi$ of $T_{\gamma, d} V$, the variety $V$ must satisfy a certain hyperbolicity condition which we call $(\gamma, d)$-hyperbolicity (see Proposition 3.9). These necessary conditions are also sufficient for (SPL) if $V$ is an algebraic curve (see Theorem 3.10). However, for the characterization of (SPL) for algebraic surfaces, we need a further condition for real singular points of $T_{\gamma, d} V$. As Proposition 3.14 shows, these singular points come in two classes. For one class $(\gamma, d)$-hyperbolicity is still the necessary condition, while the more sophisticated $(\gamma, d, \delta)$-hyperbolicity is needed for the other class (see Proposition 3.14). Collecting all these necessary conditions-except the first one-we define algebraic surfaces in $\mathbb{C}^{n}$ which are called "hyperbolic in conoids". Using this notion we show that for an algebraic surface in $\mathbb{C}^{n}$ to satisfy (SPL) it is necessary to be hyperbolic in conoids and to satisfy $\mathrm{PL}_{\text {loc }}(\xi)$ at each real point $\xi$ in $V$.

The main result of Section 4 is to prove that this necessary condition is also sufficient. In order to do so we have to overcome the difficulty that we have too many necessary conditions. Using a finiteness result from [9], we are able to single out a finite number of real simple curves $\gamma$ and exponents $d$ for which the conditions appearing in the definition of hyperbolicity in conoids are already sufficient. Then a suitable modification of the arguments which we used in [5] to characterize 
the analytic surfaces in $\mathbb{C}^{3}$ which satisfy $\mathrm{PL}_{\text {loc }}$ can be applied to prove the desired characterization (see Theorem 4.3).

In Section 5 we use the results of the previous sections to treat a number of examples. This illustrates that our methods are almost algorithmic. Example 5.6 shows that (SPL) is a very subtle property. Changing a polynomial by adding or deleting a constant may destroy (SPL) for its zero variety. We also indicate how the main result of [3] can be deduced from the characterization given in the present paper.

\section{Preliminaries}

In this section we fix the notation and recall some basic facts that are needed in the subsequent sections.

Throughout this paper, $|\cdot|$ denotes the Euclidean norm on $\mathbb{C}^{n}, B(\xi, r)$ or $B^{n}(\xi, r)$ denotes the open ball with center $\xi$ and radius in $\mathbb{C}^{n}$, and $S^{n}$ denotes the Euclidean unit sphere in $\mathbb{R}^{n+1}$.

Definition 2.1 (a) Let $V$ be the germ of an analytic variety at some point $p$ in $\mathbb{C}^{n}$. The tangent cone $T_{p} V$ of $V$ at $p$ is defined as the set of all $v \in \mathbb{C}^{n}$ which are tangent to $V$ at $p$. Here $v \in \mathbb{C}^{n}$ is tangent to $V$ at $p$ if there exist a sequence $\left(p_{j}\right)_{j \in \mathbb{N}}$ in $V$ converging to $p$ and a sequence $\left(a_{j}\right)_{j \in \mathbb{N}}$ in $\mathbb{C}$ such that $\lim _{j \rightarrow \infty} a_{j}\left(p_{j}-p\right)=v$. For a general discussion of tangent cones at $p$, see Whitney [17], Chapter 7. The tangent cone defined here is Whitney's cone $C_{3}$.

(b) For an algebraic variety $V$ in $\mathbb{C}^{n}$ its cone of limiting directions $V_{h}$ is defined as

$$
V_{h}:=\left\{r \lim _{j \rightarrow \infty} \frac{z_{j}}{\left|z_{j}\right|}: r \geq 0, z_{j} \in V,\left|z_{j}\right| \rightarrow \infty\right\} .
$$

For a different description see [4], 2.4, where it is proved in particular that $V_{h}$ is an algebraic variety.

Definition 2.2 A simple curve $\gamma$ in $\mathbb{C}^{n}$ is a map $\gamma:\left[\alpha, \infty\left[\rightarrow \mathbb{C}^{n}\right.\right.$ which for some $\alpha>0$ and some $q \in \mathbb{N}$ admits a convergent expansion

$$
\gamma(t)=\sum_{j=-\infty}^{q} \xi_{j} t^{j / q} \text { with }\left|\xi_{q}\right|=1 .
$$

The vector $\xi_{q}$ is called the limit vector of $\gamma$ at infinity. The trace of $\gamma$ is defined as $\operatorname{tr}(\gamma):=\gamma\left(\left[\alpha, \infty[)\right.\right.$. A real simple curve is a simple curve $\gamma$ satisfying $\operatorname{tr}(\gamma) \subset \mathbb{R}^{n}$.

Remark 2.3 (a) If $\gamma:\left[\alpha, \infty\left[\rightarrow \mathbb{C}^{n}\right.\right.$ is a simple curve then for some $\beta \geq \alpha$ the restriction of $\gamma$ to $[\beta, \infty$ [ is injective. Hence it is no restriction to assume that $\gamma$ is injective.

Definition 2.4 A real simple curve $\gamma$ in $\mathbb{R}^{n}$ is said to be in standard parametrization with respect to a basis $\left(\xi_{1}, \ldots, \xi_{n}\right)$ of $\mathbb{R}^{n}$ if for some $q \in \mathbb{N}$ we have $\gamma(t)=t \xi_{1}+\sum_{\nu=2}^{n} \gamma_{\nu}(t) \xi_{\nu}$, where $\gamma_{v}(t)=\sum_{j=-\infty}^{q-1} a_{v, j} t^{j / q}$.

From [7], Lemma 2.5, we recall the following lemma. 
Lemma 2.5 (a) Let $V \subset \mathbb{C}^{n}$ be a pure 1-dimensional analytic variety in $\mathbb{C}^{n}$ and let $T$ be a branch of $V \cap \mathbb{R}^{n}$ at infinity. Then there exist a basis $\left(\xi_{1}, \ldots, \xi_{n}\right)$ of $\mathbb{R}^{n}, r>0$, and a real simple curve $\gamma$ in standard parametrization such that $T \backslash B^{n}(0, r)=\operatorname{tr}(\gamma)$.

(b) Let $\gamma$ be a real simple curve in $\mathbb{R}^{n}$ and let $\left(\xi_{1}, \ldots, \xi_{n}\right)$ be a basis of $\mathbb{R}^{n}$ such that $\xi_{1}$ is the limit vector of $\gamma$ at infinity. Then there are $r>0$ and a real simple curve $\delta$ which is in standard parametrization with respect to $\left(\xi_{1}, \ldots, \xi_{n}\right)$ such that $\operatorname{tr}(\gamma) \backslash B^{n}(0, r)=\operatorname{tr}(\delta)$.

Definition 2.6 (a) Let $V \subset \mathbb{C}^{n}$ be an algebraic variety of pure dimension $k \geq 1$, let $\gamma:\left[\alpha, \infty\left[\rightarrow \mathbb{C}^{n}\right.\right.$ be a simple curve, and let $d \leq 1$. Then for $t \in[\alpha, \infty[$ we define

$$
V_{\gamma, d, t}:=\left\{w \in \mathbb{C}^{n}: \gamma(t)+w t^{d} \in V\right\}=\frac{1}{t^{d}}(V-\gamma(t))
$$

and we define the limit variety $T_{\gamma, d} V$ of $V$ of order $d$ along $\gamma$ as the set

$$
\begin{aligned}
& T_{\gamma, d} V:=\left\{\zeta \in \mathbb{C}^{n}: \zeta=\lim _{j \rightarrow \infty} z_{j}, \text { where } z_{j} \in V_{\gamma, d, t_{j}} \text { for } j \in \mathbb{N}\right. \text { and } \\
&\left(t_{j}\right)_{j \in \mathbb{N}} \text { is a sequence in }[\alpha, \infty[\text { which tends to infinity }\} .
\end{aligned}
$$

If it is clear from the context we will sometimes write $V_{d, t}$ or just $V_{t}$ instead of $V_{\gamma, d, t}$.

(b) Let $V$ be the germ of an analytic variety at some point $p$ in $\mathbb{C}^{n}$. The tangent cone $T_{p} V$ of $V$ at $p$ is defined as the set of all $v \in \mathbb{C}^{n}$ which are tangent to $V$ at $p$. Here $v \in \mathbb{C}^{n}$ is tangent to $V$ at $p$ if there exist a sequence $\left(p_{j}\right)_{j \in \mathbb{N}}$ in $V$ converging to $p$ and a sequence $\left(a_{j}\right)_{j \in \mathbb{N}}$ in $\mathbb{C}$ such that $\lim _{j \rightarrow \infty} a_{j}\left(p_{j}-p\right)=v$.

From [8] we recall the following results about limit varieties.

Theorem 2.7 Let $V$ be an algebraic variety of pure dimension $k \geq 1$ in $\mathbb{C}^{n}$, let $\gamma:\left[\alpha, \infty\left[\rightarrow \mathbb{C}^{n}\right.\right.$ be a simple curve in $\mathbb{C}^{n}$ with limit vector $\xi$ at infinity, and let $d \leq 1$ be given. Then the following assertions hold:

(a) $T_{\gamma, d} V$ is either empty or an algebraic variety of pure dimension $k$.

(b) $T_{\gamma, 1} V=V_{h}-\xi$.

(c) If $d<1$ then $w \in T_{\gamma, d} V$ if and only if $w+\lambda \xi \in T_{\gamma, d} V$ for each $\lambda \in \mathbb{C}$.

(d) For each $R>0$ there exists $\alpha_{0} \geq \alpha$ such that for each sequence $\left(t_{j}\right)_{j \in \mathbb{N}}$ in $\left[\alpha_{0}, \infty\left[\right.\right.$ which tends to infinity, the varieties $\left(V_{\gamma, d, t_{j}} \cap B(0, R)\right)_{j \in \mathbb{N}}$ converge to $T_{\gamma, d} V \cap B(0, R)$ in the sense of Meise, Taylor, and Vogt [15], 4.3.

Definition 2.8 Let $\gamma:\left[\alpha, \infty\left[\rightarrow \mathbb{R}^{n}\right.\right.$ be a real simple curve, let $d \leq 1$, a subset $U$ of $\mathbb{C}^{n}$, and $R \geq \alpha$ be given. We call

$$
\Gamma(\gamma, d, U, R):=\bigcup_{t>R}\left(\gamma(t)+t^{d} U\right)
$$

the conoid with core $\gamma$, opening exponent $d$, and profile $U$, with tip truncated at $R$. 
Definition 2.9 Two simple curves $\gamma$ and $\sigma$ in $\mathbb{C}^{n}$ are called equivalent modulo $d$ for $d \leq 1$ if for each zero neighborhood $U$ in $\mathbb{C}^{n}$ and each $R \geq 1$ we have

$$
\Gamma(\gamma, d, U, R) \cap \Gamma(\sigma, d, U, R) \neq \emptyset .
$$

If $T$ is a branch of $V \cap \mathbb{R}^{n}$ at infinity as in Lemma 2.5 then $T$ is said to be equivalent to $\gamma$ modulo $d$ if there exists a real simple curve $\sigma$ which is equivalent to $\gamma$ modulo $d$ and satisfies $\operatorname{tr}(\sigma)=T$.

Definition 2.10 Let $V$ be an analytic variety in $\mathbb{C}^{n}$ and let $\Omega$ be an open subset of $V$.

(a) By $\Omega_{\text {reg }}$ (resp. $\Omega_{\text {sing }}$ ) we denote the set of all regular (resp. singular) points of $V$ in $\Omega$.

(b) A function $u: \Omega \rightarrow[-\infty, \infty$ [ is called plurisubharmonic if it is locally bounded above, plurisubharmonic in the usual sense on $\Omega_{\text {reg }}$ and satisfies

$$
u(z)=\limsup _{\zeta \in \Omega_{\mathrm{reg}}, \zeta \rightarrow z} u(\zeta)
$$

at the singular points of $V$ in $\Omega$. By $\operatorname{PSH}(\Omega)$ we denote the set of all plurisubharmonic functions on $\Omega$.

\section{Necessary conditions}

In this section we will derive necessary conditions for an algebraic variety $V$ of pure dimension $k$ in $\mathbb{C}^{n}$ to satisfy the following condition (SPL).

Definition 3.1 An algebraic variety $V$ in $\mathbb{C}^{n}$ satisfies the condition (SPL) if there exists a constant $A \geq 1$ such that for each $u \in \operatorname{PSH}(V)$ the conditions $(\alpha)$ and $(\beta)$ imply $(\gamma)$, where

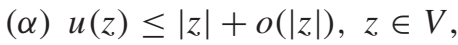

(ק) $u(z) \leq 0, z \in V \cap \mathbb{R}^{n}$,

$(\gamma) u(z) \leq A|\operatorname{Im} z|, z \in V$.

We will write $\operatorname{SPL}(A)$ when we want to specify the constant $A$.

Remark 3.2 By the classical Phragmén-Lindelöf theorem for plurisubharmonic functions on $\mathbb{C}^{n}, V=\mathbb{C}^{n}$ satisfies the condition SPL(1). Hence one can consider algebraic varieties which satisfy (SPL) as those for which the classical PhragménLindelöf theorem holds. In [3], Proposition 2.8, we pointed out why it would be too restrictive to require $A=1$ in Definition 3.1.

Besides this interpretation the property (SPL) also plays a role in the characterization of those polynomials $P \in \mathbb{C}\left[z_{1}, \ldots, z_{n}\right]$ for which the differential operator $P(D): \mathcal{D}^{\prime}\left(\mathbb{R}^{n}\right) \rightarrow \mathcal{D}^{\prime}\left(\mathbb{R}^{n}\right)$ admits a continuous linear right inverse, as it was shown in Meise and Taylor [14], Theorem 3.4, and in [3].

Remark A weaker condition than (SPL) is obtained if in Definition 3.1 one requires the existence of $A, B>0$ such that for each $u \in \operatorname{PSH}(V)$ the conditions $(\alpha)$ and $(\beta)$ in 3.1 imply 
$\left(\gamma^{\prime}\right) u(z) \leq A|\operatorname{Im} z|+B, z \in V$.

This property was called $\operatorname{SPL}(A, B)$ in Meise and Taylor [14], Definition 3.1. The algebraic surfaces $V$ in $\mathbb{C}^{3}$ which satisfy $\operatorname{SPL}(A, B)$ for some $A, B>0$ were characterized in [7], Theorem 4.7, since one can take $\omega \equiv 1$ in that theorem.

To derive necessary conditions for (SPL), we recall the definition of the local Phragmén-Lindelöf condition that was used by Hörmander [12] to characterize those differential operators $P(D)$ that are surjective on the space $\mathcal{A}\left(\mathbb{R}^{n}\right)$ of all real analytic functions on $\mathbb{R}^{n}$.

Definition 3.3 For $\xi \in \mathbb{R}^{n}$ and $r_{0}>0$ let $V$ be an analytic variety in $B\left(\xi, r_{0}\right)$ which contains $\xi$. We say that $V$ satisfies the condition $\mathrm{PL}_{\text {loc }}(\xi)$ if there exist positive numbers $A$ and $r_{0} \geq r_{1} \geq r_{2}$ such that each $u \in \operatorname{PSH}\left(V \cap B\left(\xi, r_{1}\right)\right)$ satisfying

( $\alpha) u(z) \leq 1, z \in V \cap B\left(\xi, r_{1}\right)$ and

( $\beta) u(z) \leq 0, z \in V \cap \mathbb{R}^{n} \cap B\left(\xi, r_{1}\right)$

also satisfies

$(\gamma) u(z) \leq A|\operatorname{Im} z|, z \in V \cap B\left(\xi, r_{2}\right)$.

For other equivalent definitions of $\mathrm{PL}_{\mathrm{loc}}(\xi)$ we refer to [5], Lemma 3.3.

From Meise and Taylor [14], Proposition 4.4, we recall the following result.

Proposition 3.4 If an algebraic variety $V$ in $\mathbb{C}^{n}$ satisfies (SPL) then for each $\xi \in V \cap \mathbb{R}^{n}$ it satisfies $\operatorname{PL}_{\text {loc }}(\xi)$.

Since by [7], Lemma 3.2, the condition (SPL) is equivalent to the condition $\operatorname{PL}(\omega)$ for $\omega \equiv 0$, we get from [7], Theorem 3.12, the following necessary condition for (SPL).

Proposition 3.5 Let $V$ be an algebraic variety of pure dimension $k \geq 1$ in $\mathbb{C}^{n}$ which satisfies (SPL). Then for each real simple curve $\gamma$ and each $d \in]-\infty, 1]$, the limit variety $T_{\gamma, d} V$ satisfies (SPL). In particular, $V_{h}$ satisfies (SPL).

In order to state further necessary conditions for (SPL) we introduce the following notions:

Definition 3.6 Let $V$ be an analytic variety in a neighborhood of a point $\xi \in V \cap \mathbb{R}^{n}$. We say that $V$ satisfies the dimension condition at $\xi$ if for each locally irreducible component $W$ of $V$ at $\xi$, the dimension of $W \cap \mathbb{R}^{n}$ as a real analytic variety is equal to the dimension of $W$ at $\xi$ as a complex variety.

Remark If $V$ satisfies $\mathrm{PL}_{\mathrm{loc}}(\xi)$ at $\xi \in V \cap \mathbb{R}^{n}$, then $V$ satisfies the dimension condition at $\xi$. This follows from Meise, Taylor, and Vogt [15], Lemma 2.8, since the condition $\mathrm{PL}_{\mathrm{loc}}(\xi)$ implies the condition $\mathrm{RPL}_{\mathrm{loc}}(\xi)$, defined in [15], 2.3.

Definition 3.7 (a) Let $V$ be an analytic variety in $\mathbb{C}^{n}$ which is of pure dimension $k \geq 1$ in $\zeta \in V$. A projection $\pi: \mathbb{C}^{n} \rightarrow \mathbb{C}^{n}$ is called noncharacteristic for $V$ at $\zeta$ if its rank is $k$, its image and its kernel are spanned by real vectors, and $T_{\zeta} V \cap \operatorname{ker} \pi=\{0\}$. 
(b) Let $V$ be an algebraic variety in $\mathbb{C}^{n}$ of pure dimension $k \geq 1$. A projection $\pi: \mathbb{C}^{n} \rightarrow \mathbb{C}^{n}$ is called noncharacteristic for $V$ at infinity if its rank is $k$, its image and its kernel are spanned by real vectors, and $V_{h} \cap \operatorname{ker} \pi=\{0\}$.

Hence a projection is noncharacteristic for $V$ at $\zeta$ if its kernel is transverse to $V$ at $\zeta$ and its image and kernel are spanned by real vectors.

Definition 3.8 Let $V$ be an algebraic variety of pure dimension $k \geq 1$ in $\mathbb{C}^{n}$, let $\gamma:\left[\alpha, \infty\left[\rightarrow \mathbb{C}^{n}\right.\right.$ be a real simple curve, let $d \leq 1$, and let $\zeta \in T_{\gamma, d} V \cap \mathbb{R}^{n}$. We say that $V$ is $(\gamma, d)$-hyperbolic at $\zeta$ with respect to a projection $\pi: \mathbb{C}^{n} \rightarrow \mathbb{C}^{n}$ which is noncharacteristic for $T_{\gamma, d} V$ at $\zeta$ if there exist a zero neighborhood $U$ in $\mathbb{C}^{n}$ and $r>\alpha$ such that $z \in V \cap \Gamma(\gamma, d, \zeta+U, r)$ is real whenever $\pi(z)$ is real. $V$ is called $(\gamma, d)$-hyperbolic at $\zeta$ if it is $(\gamma, d)$-hyperbolic at $\zeta$ with respect to some projection $\pi$ as above.

From Proposition 3.5 and [7], Lemma 3.20, applied with $\omega \equiv 0$ we get that also the following condition is necessary for (SPL).

Proposition 3.9 Let $V \subset \mathbb{C}^{n}$ be a pure $k$-dimensional algebraic variety in $\mathbb{C}^{n}$ which satisfies (SPL). Then for each real simple curve $\gamma:\left[\alpha, \infty\left[\rightarrow \mathbb{C}^{n}\right.\right.$, each $d \leq 1$, each $\xi \in\left(T_{\gamma, d} V\right)_{\text {reg }} \cap \mathbb{R}^{n}$, and each projection $\pi: \mathbb{C}^{n} \rightarrow \mathbb{C}^{n}$ which is noncharacteristic for $T_{\gamma, d} V$ at $\xi, V$ is $(\gamma, d)$-hyperbolic at $\xi$ with respect to $\pi$.

The necessary conditions which we found so far lead to the following characterization of the algebraic curves in $\mathbb{C}^{n}$ that satisfy (SPL).

Theorem 3.10 For each algebraic curve $V$ in $\mathbb{C}^{n}$ the following conditions are equivalent:

(a) V satisfies (SPL).

(b) $V$ satisfies $\mathrm{PL}_{\mathrm{loc}}(\xi)$ for each $\xi \in V \cap \mathbb{R}^{n}$, $V_{h}$ satisfies (SPL), and for each $\eta \in V_{h} \cap S^{n-1}$ and $\gamma_{\eta}: t \mapsto \eta t, t \geq 1, V$ is $\left(\gamma_{\eta}, 1\right)$-hyperbolic at $0 \in T_{\gamma_{\eta}, 1} V$.

(c) $V$ satisfies $\mathrm{PL}_{\mathrm{loc}}(\xi)$ for each $\xi \in V \cap \mathbb{R}^{n}, V_{h}$ satisfies (SPL), and for each (some) projection $\pi: \mathbb{C}^{n} \rightarrow \mathbb{C}^{n}$ which is noncharacteristic for $V_{h}$ at infinity with $\operatorname{im} \pi=\zeta \mathbb{C}$ for some $\zeta \in \mathbb{R}^{n}$, there exists $K \geq 1$ such that $\pi^{-1}(t \zeta) \cap V \subset$ $\mathbb{R}^{n}$ for each $t \in \mathbb{R},|t| \geq K$.

Proof (a) $\Rightarrow$ (b): By Proposition 3.4, the first condition in (b) holds. Also the second one holds, since $V_{h}$ satisfies (SPL) by Proposition 3.5. To show the third one, note that $V_{h}$ is homogeneous of dimension 1 (see [4], 2.4), hence a finite union of lines through the origin. Since $V_{h}$ satisfies (SPL) and hence $\mathrm{PL}_{\text {loc }}(0)$ by Proposition 3.4, it follows from [5], Proposition 3.16, that these lines admit real generators, i.e., there are $\mu \in \mathbb{N}$ and $a_{j} \in \mathbb{R}^{n},\left|a_{j}\right|=1,1 \leq j \leq \mu$, such that

$$
V_{h}=\bigcup_{j=1}^{\mu} a_{j} \cdot \mathbb{C} .
$$

In particular, each point $\eta \in V_{h} \cap S^{n-1}$ is a regular point of $V_{h}$. By Theorem 2.7 we have $T_{\gamma_{\eta}, 1} V=V_{h}-\eta$. Hence 0 is a regular point of $T_{\gamma_{\eta}, 1} V$. Therefore, the third condition in (b) follows from Proposition 3.9.

(b) $\Rightarrow$ (a) and (b) $\Leftrightarrow$ (c): This is proved in Remark 4.13 since later we have to use the same arguments in greater generality. 
Remark Note that an analytic curve $V$ in $\mathbb{C}^{n}$ satisfies $\mathrm{PL}_{\text {loc }}(\xi)$ at some $\xi \in V \cap \mathbb{R}^{n}$ if and only if in suitable coordinates and in some neighborhood of $\xi, V$ is the union of graphs of holomorphic maps which are real over real points. This follows from [5], Proposition 3.16.

As a corollary we obtain the following characterization of the algebraic curves in $\mathbb{C}^{n}$ satisfying (SPL), which was proved in [6], Proposition 18.

Corollary 3.11 Let $P \in \mathbb{C}[x, y]$ be of degree $m>0$. Then $V:=V(P)$ satisfies the condition (SPL) if and only if the following two conditions are satisfied:

(a) $V$ satisfies $\mathrm{PL}_{\mathrm{loc}}(\xi)$ at each $\xi \in V \cap \mathbb{R}^{2}$.

(b) For each (or some) $\eta, \zeta \in S^{1}$ satisfying $P_{m}(\zeta) \neq 0$ and $\operatorname{span}_{\mathbb{R}}(\eta, \zeta)=\mathbb{R}^{2}$ there exists $R>0$ such that for each $t \in \mathbb{R},|t| \geq R$, the polynomial $\lambda \mapsto$ $P(t \eta+\lambda \zeta)$ has only real zeros.

Proof If $V(P)$ satisfies (SPL) then Theorem 3.10 implies (a). As in the proof of Theorem 3.10 there exist $\mu \in \mathbb{N}$ and $a_{j}=\left(\alpha_{j}, \beta_{j}\right) \in \mathbb{R}^{2} 1 \leq j \leq \mu$, so that (3.1) holds. Now fix $\eta, \zeta \in S^{1}$ as in (b). Then the projection $\pi: \mathbb{C}^{2} \rightarrow \mathbb{C}^{2}$, $\pi(a \eta+b \zeta):=a \eta$ is noncharacteristic for $V_{h}$ at infinity. Hence there exists $S>0$ such that $\left.\pi\right|_{V(P)}$ is proper and unbranched over $\mathbb{C} \backslash B(0, S)$. In particular, for each $t \in \mathbb{R},|t|>S$, the set $\pi^{-1}(t \eta)$ has $m$ elements. Because of the hyperbolicity condition in 3.10(b), all these points must be real. Hence (b) holds.

If the conditions (a) and (b) hold for some $\eta, \zeta \in S^{1}$ then it is easy to check that $V(P)$ satisfies the conditions in 3.10(b). To obtain the statement about $V_{h}$, note that $V_{h}$ is a finite union of complex lines in $\mathbb{C}^{2}$. They must have real generators because otherwise condition (b) cannot hold.

Remark (a) In Example 5.1 we define an irreducible polynomial $P \in \mathbb{C}[x, y]$ for which $V(P)$ satisfies (SPL), while the principal part of $P$ is not square-free. Hence the number $\mu$ in (3.1) can be smaller than the number of branches in $V$. This shows that the general situation, even for $n=2$, differs from the special one that was treated in Meise and Taylor [14], Corollary 4.10.

(b) Example 5.2 shows that $V(P) \cap \mathbb{R}^{2}$ can have bounded connected components, while $V(P)$ satisfies (SPL).

Definition 3.12 Let $V$ be an algebraic variety in $\mathbb{C}^{n}$ of pure dimension $k \geq 1$ and let $\gamma$ be a real simple curve. Then it was shown in [8], Proposition 5 and [9], 4.9 , that there exist $p \in \mathbb{N}$ and rational numbers $1=d_{1}>\ldots>d_{p}$ such that $d \mapsto T_{\gamma, d} V$ is constant on the intervals $] d_{j+1}, d_{j}[, 1 \leq j \leq p-1$ and $]-\infty, d_{p}$ [ and that these numbers are minimal in this respect. We call them the critical values for the curve $\gamma$ and the variety $V$.

Let $d$ be a critical value for $\gamma$ and $V$. A singular point $\zeta$ of $T_{\gamma, d} V \cap \mathbb{R}^{n}$ is said to be terminating for $\gamma$ and $d$ if there is a simple curve $\sigma(t)=\gamma(t)+\zeta t^{d}+o\left(t^{d}\right)$ such that no critical value for $\sigma$ and $V$ is smaller than $d$. A point $\zeta_{1} \in\left(V_{h}\right)_{\text {sing }}$ with $\left|\zeta_{1}\right|=1$ is called terminating if 0 is terminating for $\gamma_{1}(t):=t \zeta_{1}$ and $d=1$.

If $d \leq 1$ is a critical value for $\gamma$ and if $\zeta \in\left(T_{\gamma, d} V\right)_{\text {sing }} \cap \mathbb{R}^{n}$ is not terminating for $\gamma$ and $d$, then $\Delta(\gamma, d, \zeta)$ denotes the largest critical value for $\gamma_{\zeta}: t \mapsto \gamma(t)+\zeta t^{d}$ which is smaller than $d$. 
Definition 3.13 (a) Let $V$ be an algebraic variety in $\mathbb{C}^{n}$ of pure dimension $k \geq 1$, let $\gamma$ be a real simple curve, and let $d \leq 1$ be a critical value for $\gamma$ and $V$. Assume that $\xi \in T_{\gamma, d} V \cap \mathbb{R}^{n}$ and let $\delta<d \leq 1$. Then we say that $V$ is $(\gamma, d, \delta)$-hyperbolic at $\xi$ with respect to a projection $\pi$ which is noncharacteristic for $T_{\gamma, d} V$ at $\xi$ if there exist $R>1$ and zero neighborhoods $U \subset B\left(0, \frac{1}{2}\right)$ and $G \subset \mathbb{C}^{n}$ bounded, such that for each $\zeta \in V \cap \Gamma(\gamma, d, \xi+U, R)$ for which $\pi(\zeta)$ is real and $\pi(\zeta) \notin \Gamma(\pi \circ \gamma, \delta, \pi(\xi)+\pi(G), 0)$ also $\zeta$ is real.

(b) Let $W$ be an algebraic surface in $\mathbb{C}^{n}$, denote by $\pi: \mathbb{C}^{n} \rightarrow \mathbb{C}^{n}$ the projection $\pi\left(z^{\prime}, z_{n-1}, z_{n}\right)=\left(0, z_{n-1}, z_{n}\right)$ and let $U$ be a subset of $\mathbb{C}^{n}$. Then $W \cap U$ is called hyperbolic with respect to $\pi$ if $\zeta \in W \cap U$ is real whenever $\pi(\zeta)$ is real.

Proposition 3.14 Let $V$ be an algebraic surface in $\mathbb{C}^{n}$ that satisfies (SPL), let $\gamma$ be a real simple curve which is in standard parametrization with respect to the last variable, and let $d \leq 1$ be a critical value for $\gamma$ and $V$. Assume further that $0 \in$ $\left(T_{\gamma, d} V\right)_{\text {sing }}$ and that the projection $\pi: \mathbb{C}^{n} \rightarrow \mathbb{C}^{n}, \pi\left(z^{\prime}, z_{n-1}, z_{n}\right):=\left(0, z_{n-1}, z_{n}\right)$ is noncharacteristic for $T_{\gamma, d} V$ at zero. Then the following assertions hold:

(a) If $0 \in T_{\gamma, d} V$ is not terminating for $\gamma$ and $d$ then $V$ is $(\gamma, d, \delta)$-hyperbolic at 0 with respect to $\pi$ for $\delta:=\Delta(\gamma, d, 0)$.

(b) If $0 \in T_{\gamma, d} V$ is terminating for $\gamma$ and $d$ then $V$ is $(\gamma, d)$-hyperbolic at $0 \in$ $T_{\gamma, d} V$.

Proof (a) We first treat the case that $d<1$ and that 0 is not terminating for $\gamma$ and $d$. Since $d$ is less then 1, it follows from Theorem 2.7 (c) that there are algebraic curves $W_{d}$ and $W_{\delta}$ satisfying $T_{\gamma, d} V=W_{d} \times \mathbb{C}$ and $T_{\gamma, \delta} V=W_{\delta} \times \mathbb{C}$. By Proposition 3.5 , the hypothesis implies that $T_{\gamma, d} V$ and $T_{\gamma, \delta} V$ satisfy (SPL). Hence $W_{d}$ and $W_{\delta}$ satisfy (SPL). By Proposition 3.4, $W_{d}$ satisfies $\mathrm{PL}_{\mathrm{loc}}(0)$. Hence [5], Proposition 3.16 , implies the existence of $\varepsilon_{1}, \varepsilon_{2}>0$ such that the following conditions are satisfied:

(i) $T_{\gamma, d} V \cap\left(B^{n-2}\left(0, \varepsilon_{1}\right) \times B^{1}\left(0, \varepsilon_{2}\right) \times \mathbb{C}\right)$ is hyperbolic with respect to $\pi$.

(ii) $\pi$ restricted to $T_{\gamma, d} V \cap\left(B^{n-2}\left(0, \varepsilon_{1}\right) \times\left(B^{1}\left(0,2 \varepsilon_{2}\right) \backslash\{0\}\right) \times \mathbb{C}\right)$ is unbranched.

(iii) If $w \in T_{\gamma, d}$ satisfies $\left|w_{n-1}\right| \leq 2 \varepsilon_{2}$ then $\left|w^{\prime}\right| \leq \varepsilon_{1}$ for $w=\left(w^{\prime}, w_{n-1}, w_{n}\right)$.

Next note that by [9], Corollary 4.15, we have $\left(T_{\gamma, \delta} V\right)_{h}=T_{0}\left(T_{\gamma, d} V\right)$. Hence the assumption on $\pi$ implies that $\pi$ is noncharacteristic for $\left(T_{\gamma, \delta} V\right)_{h}$ at infinity. Since $T_{\gamma, \delta} V$ satisfies (SPL), it follows from Theorem 3.10 (applied to $W_{\delta}$ ) that we can choose $K_{1}, K_{2}>1$ such that the following conditions are satisfied:

(iv) $T_{\gamma, \delta} V \backslash\left(\mathbb{C}^{n-2} \times B^{1}\left(0, K_{2}\right) \times \mathbb{C}\right)$ is hyperbolic with respect to $\pi$.

(iiv) $\pi$ restricted to $T_{\gamma, \delta} V \backslash\left(\mathbb{C}^{n-2} \times B^{1}\left(0, K_{2}\right) \times \mathbb{C}\right)$ is unbranched.

(iiiv) If $w \in T_{\gamma, \delta} V$ satisfies $\left|w_{n-1}\right| \leq 2 K_{2}$ then $\left|w^{\prime}\right| \leq K_{1}$.

Next let

$$
\begin{aligned}
& \Gamma_{R}:=\Gamma\left(\gamma, d, B^{n-2}\left(0, \varepsilon_{1}\right) \times B^{2}\left(0, \varepsilon_{2}\right), R\right), R>1, \\
& \Gamma_{R}^{\prime}:=\Gamma\left(\gamma, \delta, B^{n-2}\left(0, K_{1}\right) \times B^{2}\left(0,2 K_{2}\right), R\right), R>1,
\end{aligned}
$$

and define

$$
M_{R}:=V \cap\left(\mathbb{C}^{n-2} \times \mathbb{R}^{2}\right) \cap \bar{\Gamma}_{R} \backslash \Gamma_{R}^{\prime} .
$$


For $R \geq R_{1}, M_{R}$ is a semi-algebraic set. Moreover, the function

$h:\left[R_{1}, \infty\left[\rightarrow R, h(r):=\sup \left\{\left|\operatorname{Im} x^{\prime}\right|:\right.\right.\right.$ there is $y \in \mathbb{R}$ with $\left.\left(x^{\prime}, y, r\right) \in M_{R}\right\}$

is semi-algebraic. Hence the curve selection theorem (see e.g. Hörmander [13], Theorem A.2.8) implies the existence of $R_{2} \geq R_{1}$ and of an algebraic curve $\sigma: \quad\left[R_{2}, \infty\left[\rightarrow M_{R}\right.\right.$ which satisfies $\pi_{n}(\sigma(r))=r$ and $h(r)=\left|\operatorname{Im} \sigma^{\prime}(r)\right|$. Now we distinguish two cases: case 1: $h(r) \equiv 0$ for all large $r$. In this case the Proposition is proved.

case 2: $h(r) \not \equiv 0$ for all large $r$.

Since $h$ is a semi-algebraic function, the hypothesis in the present case implies the existence of $c_{1}>0, a \in \mathbb{Q}$, and $t_{0}>1$ such that

$$
h(t)=c_{1} t^{a}+o\left(t^{a}\right), t \geq t_{0} .
$$

Next note that we can choose $t_{0}$ so large that the curve $\sigma$ has a convergent Puiseux series expansion of the form

$$
\sigma(t)=\gamma(t)+\left(t^{b} w^{\prime}, t^{b} w_{n-1}, 0\right)+\tilde{\sigma}(t), t \geq t_{0},
$$

where $\tilde{\sigma}$ satisfies $\pi_{u} \circ \tilde{\sigma} \equiv 0$ and $|\tilde{\sigma}(t)|=o\left(t^{b}\right)$ as $t$ tends to infinity. Since $\operatorname{tr}(\sigma) \subset M_{R}$, the number $b \in \mathbb{Q}$ must satisfy $\delta \leq b \leq d$. From

$$
h(t)=\left|\operatorname{Im} \sigma^{\prime}(t)\right| \leq\left|\sigma^{\prime}(t)\right|=O\left(t^{b}\right), t \geq t_{0},
$$

we conclude $a \leq b$.

Now we can apply the arguments of the proof of [6], Proposition 20, with $\omega \equiv 0$ to conclude that $h(t) \equiv 0$, in contradiction to the present hypothesis. Hence case 2 is excluded and part (a) of the Proposition is proved for $d<1$.

If $d=1$ then the arguments of the proof have to be modified since $T_{\gamma, 1} V$ is not necessarily the product of a curve in $\mathbb{C}^{2}$ and $\mathbb{C}$. However, it is the translate of a homogeneous two-dimensional algebraic variety. Therefore, almost the same arguments as for $d<1$ apply.

(b) If $0 \in T_{\gamma, d} V$ is terminating for $\gamma$ and $d$, then there exists a real simple curve $\sigma$ which is equivalent modulo $d$ to $\gamma$ so that for any $\delta, \delta^{\prime}<d$ we have $T_{\sigma, \delta} V=T_{\sigma, \delta^{\prime}} V$ and this variety is homogeneous. Obviously $T_{\gamma, d} V=T_{\sigma, d} V$, and by [9], Corollary 4.15, we have for $\delta<d$

$$
T_{\sigma, \delta} V=\left(T_{\sigma, \delta} V\right)_{h}=T_{0}\left(T_{\sigma, d} V\right)=T_{0}\left(T_{\gamma, d} V\right) .
$$

Hence the hypothesis on $\pi$ implies that $\pi$ is noncharacteristic for $T_{\sigma, \delta} V$ at infinity. Since $V$ satisfies (SPL), Proposition 3.5 implies that $T_{\sigma, \delta} V$ satisfies (SPL). Because of $\delta<d \leq 1$ we get from Theorem $2.7(\mathrm{~d})$, that $T_{\sigma, \delta} V=W \times \mathbb{C}$ for some algebraic curve in $\mathbb{C}^{n-1}$, which also satisfies (SPL) and which is homogeneous. Hence it follows from [5], Proposition 3.16, that for some $k \in \mathbb{N}$ and $a_{1}, \ldots, a_{k} \in \mathbb{R}^{n-1}$ we have

$$
W=\bigcup_{j=1}^{k} \mathbb{C} a_{j} .
$$


If $d<1$ then we get as in part (a) that $T_{\sigma, d} V=T_{\gamma, d} V=W_{d} \times \mathbb{C}$ and that there are $\varepsilon_{1}, \varepsilon_{2}>0$ such that (i)-(iii) of part (a) hold with $T_{\gamma, d} V$ replaced by $T_{\sigma, d} V$. For fixed $\delta<d$ the preceding considerations show that $T_{\sigma, \delta} V$ is hyperbolic with respect to $\pi$. For $R>1$ we now let

$$
\Gamma_{R}:=\Gamma\left(\sigma, d, B^{n-1}\left(0, \varepsilon_{1}\right) \times B^{2}\left(0, \varepsilon_{2}\right), R\right)
$$

and

$$
M_{R}:=V \cap \bar{\Gamma}_{R}
$$

Then we argue as in part (a) to conclude that for each $\zeta \in V \cap \Gamma_{R}$ for which $\pi(\zeta)$ is real, also $\zeta$ is real. Hence $V$ is $(\sigma, d)$-hyperbolic at $0 \in T_{\sigma, d} V=T_{\gamma, d} V$. Since $|\sigma(t)-\gamma(t)|=o\left(t^{d}\right)$, this implies that $V$ is also $(\gamma, d)$-hyperbolic at $0 \in T_{\gamma, d} V$. If $d=1$, the proof has to be modified slightly, since $T_{\gamma, 1} V$ need not be of the form $W_{1} \times \mathbb{C}$. As in part (a) we leave this modification to the reader.

Definition 3.15 Let $V$ be an algebraic surface in $\mathbb{C}^{n}$. We say that $V$ is hyperbolic in conoids if for each real simple curve $\gamma$ and each $d \leq 1$ the following conditions are satisfied:

(1) $T_{\gamma, d} V$ satisfies (SPL).

(2) For each $\xi \in\left(T_{\gamma, d} V\right)_{\text {reg }} \cap \mathbb{R}^{n}$ and each $\xi \in\left(T_{\gamma, d} V\right)_{\text {sing }} \cap \mathbb{R}^{n}$ which is terminating for $\gamma$ and $d, V$ is $(\gamma, d)$-hyperbolic at $\xi$.

(3) For each $\xi \in\left(T_{\gamma, d} V\right)_{\text {sing }} \cap \mathbb{R}^{n}$ which is not terminating for $\gamma$ and $d, V$ is $(\gamma, d, \delta)$-hyperbolic at $\xi$ for $\delta:=\Delta(\gamma, d, \xi)$.

Remark If $V$ is an algebraic surface in $\mathbb{C}^{n}$ which satisfies $\mathrm{PL}_{\text {loc }}(\xi)$ at each $\xi \in$ $V \cap \mathbb{R}^{n}$ then condition (1) in Definition 3.15 can be weakened and we still get equivalence of $V$ being hyperbolic in conoids. For the precise formulation we refer to Corollary 4.4.

Combining the Propositions 3.5 and 3.14, we get the following theorem.

Theorem 3.16 Let $V$ be an algebraic surface in $\mathbb{C}^{n}$. If $V$ satisfies (SPL) then $V$ satisfies the following conditions:

(a) $V$ satisfies $\mathrm{PL}_{\mathrm{loc}}(\xi)$ at each $\xi \in V \cap \mathbb{R}^{n}$.

(b) $V$ is hyperbolic in conoids.

To show that these necessary conditions are in fact sufficient for algebraic curves in $\mathbb{C}^{n}$, we need some preparation which is given in the next section. We conclude this section by providing two rather coarse but useful necessary conditions for (SPL).

Lemma 3.17 Let $V$ be an algebraic variety in $\mathbb{C}^{n}$ of pure dimension $k \geq 1$ which satisfies (SPL). Then the following assertions hold:

(a) $W \cap \mathbb{R}^{n} \neq \emptyset$ for each irreducible component $W$ of $V$.

(b) The ideal $I(V):=\left\{p \in \mathbb{C}\left[z_{1} \ldots z_{n}\right]:\left.p\right|_{V} \equiv 0\right\}$ is generated by polynomials with real coefficients. 
Proof (a) This can be shown by the same arguments that were used in the proof of [7], Cor. 3.15(b).

(b) Because of (a) and Proposition 3.14 this follows from [5], Lemma 3.17.

Remark Note that an algebraic variety $V$ in $\mathbb{C}^{n}$ of pure dimension $k \geq 1$ satisfies (SPL) if and only if each irreducible component $W$ of $V$ has this property.

\section{Sufficient conditions}

To show that the necessary conditions in Theorem 3.16 are in fact sufficient for (SPL) for algebraic surfaces $V$ in $\mathbb{C}^{n}$, we have to find out which of the many necessary conditions are really needed to prove that $V$ satisfies (SPL). To do so we recall from [9], 4.16, the following definition.

Definition 4.1 Let $V$ be an algebraic variety in $\mathbb{C}^{n}$ that is of pure dimension $k$. A set $C:=\left(\zeta_{j}, d_{j}\right)_{j=1}^{l}$ in $\left(\mathbb{R}^{n} \times \mathbb{Q}\right)^{l}$ is called a critical set for $V$ of length $l=l(C)$ if the following conditions are satisfied:

(a) $\zeta_{1} \in\left(V_{h}\right)_{\text {sing }} \cap \mathbb{R}^{n},\left|\zeta_{1}\right|=1$, and $d_{1}=1$.

(b) For $1 \leq i \leq l$ define $\gamma_{i}(t):=\sum_{j=1}^{i} \zeta_{j} t^{d_{j}}$. Then for $1 \leq i \leq l-1$ we have

(b1) $d_{i+1}$ is the largest critical value for $V$ and $\gamma_{i}(t)$ strictly smaller than $d_{i}$, and

(b2) $\zeta_{i+1}$ is a real singlar point of $T_{\gamma_{i}, d_{i+1}} V$ and $\left\langle\zeta_{i+1}, \zeta_{1}\right\rangle=0$.

A critical set $C$ as above is called a normal critical set for $V$ if $\zeta_{1}$ is not terminating and if $\zeta_{i}$ is not terminating for $\gamma_{i-1}$ and $d_{i}, 2 \leq i \leq l$.

Definition 4.2 (a) For an algebraic surface $V$ in $\mathbb{C}^{n}$ we let

$$
M_{0}:=\left\{\xi \in\left(V_{h}\right)_{\operatorname{sing}} \cap S^{n-1}: \xi \text { is not terminating }\right\},
$$

and we define the relevant set $\mathcal{R}(V)$ for $V$ by

$$
\begin{array}{r}
\mathcal{R}(V):=\left\{C: C=\left(\zeta_{j}, d_{j}\right)_{j=1}^{l} \text { is a normal critical set for } V\right. \\
\text { satisfying } \left.l(C) \geq 2 \text { and } \zeta_{1} \in M_{0}\right\} .
\end{array}
$$

Of course, $M_{0}=\emptyset$ implies $\mathcal{R}(V)=\emptyset$. Note that $\mathcal{R}(V)$ is a finite set by [9], Theorem 4.18.

(b) For $C \in \mathcal{R}(V), C=\left(\zeta_{j}, d_{j}\right)_{j=1}^{l}$, we define the associated curve $\gamma_{C}$ by $\gamma_{C}(t):=\sum_{j=1}^{l-1} \zeta_{j} t^{d_{j}}$ and the associated triple by $\left(\gamma_{C}, d_{l-1}, d_{l}\right)$. Moreover, we let

$$
\mathcal{K}(V):=\left\{\left(\gamma_{C}, d_{l-1}, d_{l}\right): C=\left(\zeta_{j}, d_{j}\right)_{j=1}^{l} \in \mathcal{R}(V)\right\}
$$

and call it the set of all relevant triples for $V$.

In this section we will prove the following theorem. 
Theorem 4.3 For each algebraic surface $V$ in $\mathbb{C}^{n}$ the following conditions are equivalent:

(a) V satisfies (SPL).

(b) $V$ is hyperbolic in conoids and $V$ satisfies $\mathrm{PL}_{\mathrm{loc}}(\xi)$ at each $\xi \in V \cap \mathbb{R}^{n}$.

(c) The following conditions are satisfied:

(1) $V$ satisfies $\mathrm{PL}_{\mathrm{loc}}(\xi)$ at each $\xi \in V \cap \mathbb{R}^{n}$.

(2) $V_{h}$ satisfies (SPL).

(3) For each $\zeta \in V_{h} \cap S^{n-1}$ which is regular or a terminating singularity, $V$ is $\left(\gamma_{\zeta}, 1\right)$-hyperbolic at $0 \in T_{\gamma_{\zeta}, 1} V$ for $\gamma_{\zeta}: t \mapsto t \zeta$.

(4) For each $(\gamma, d, \delta) \in \mathcal{K}(V)$ the variety $V$ is $(\gamma, d, \delta)$-hyperbolic at $0 \in$ $T_{\gamma, d} V$ and for each $\zeta \in T_{\gamma, \delta} V \cap \mathbb{R}^{n}$ which is regular or a terminating singularity for $\gamma$ and $\delta$, the variety $V$ is $(\gamma, \delta)$-hyperbolic at $\zeta$.

Corollary 4.4 Let $V$ be an algebraic surface in $\mathbb{C}^{n}$ which satisfies $\mathrm{PL}_{\mathrm{loc}}(\xi)$ for each $\xi \in V \cap \mathbb{R}^{n}$. Then in Definition 3.15 condition (1) can be replaced equivalently by one of the following conditions:

(1') $T_{\gamma, d} V$ satisfies $\mathrm{PL}_{\mathrm{loc}}(\xi)$ at each $\xi \in T_{\gamma, d} V \cap \mathbb{R}^{n}$.

(1") $V_{h}$ satisfies (SPL) or $\mathrm{PL}_{\mathrm{loc}}(0)$.

Proof If $T_{\gamma, d} V$ satisfies (SPL) then it satisfies $\mathrm{PL}_{\text {loc }}(\xi)$ at each $\xi \in\left(T_{\gamma, d} V\right) \cap \mathbb{R}^{n}$ by Proposition 3.4. Hence condition 3.15 (1) implies condition (1').

To show that (1') implies (1"), note first that for any $\zeta \in V_{h} \cap \mathbb{R}^{n},|\zeta|=1$, and $\gamma(t):=t \zeta$, we have $T_{\gamma, 1} V=V_{h}-\zeta$ by Theorem 2.7 (b). By (1') this implies that $V_{h}$ satisfies $\mathrm{PL}_{\mathrm{loc}}(0)$. Since $V_{h}$ is homogeneous, [7], Remark 3.14, shows that $V_{h}$ satisfies (SPL). Hence (1") is satisfied.

If condition (1") and the conditions 3.15 (2) and (3) are fulfilled, then the conditions in Theorem 4.3 (c) are satisfied. Hence $V$ is hyperbolic in conoids by this theorem. In particular, condition 3.15 (1) holds.

In order to prove Theorem 4.3 we need some preparation. We begin with the following definition which coincides with [7], Definition 5.1, for $\omega$ being identically zero.

Definition 4.5 Let $V$ be an algebraic variety in $\mathbb{C}^{n}, \gamma$ a real simple curve in $\mathbb{C}^{n}$, $d \leq 1, G$ a bounded open set in $\mathbb{C}^{n}$ so that $\Gamma:=\Gamma(\gamma, d, G, R)$ is a conoid for some $R>1$ and fix a compact subset $K$ of $G$. We say that $V$ satisfies the conoidal Phragmén-Lindelöf condition $\operatorname{PL}(V, \Gamma(\gamma, d, G, K, R))$ if there exist $A_{1} \geq 1$ and $R_{1} \geq R$ such that for each $u \in \operatorname{PSH}(V \cap \Gamma)$ the following two conditions

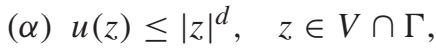

(в) $u(z) \leq 0, \quad z \in V \cap \Gamma \cap \mathbb{R}^{n}$,

imply

$(\gamma) u(z) \leq A_{1}|\operatorname{Im} z|, \quad z \in V \cap \Gamma\left(\gamma, d, K, R_{1}\right)$.

We say that $V$ satisfies $\operatorname{PL}(V, \Gamma(\gamma, d, G, R))$ if $G$ is a zero neighborhood and if $V$ satisfies $\operatorname{PL}(V, \Gamma(\gamma, d, G, K, R))$ for a suitable compact zero neighborhood $K \subset G$.

The following two lemmas are just specializations of the Lemmas 5.2 and 5.3 in [7] for $\omega \equiv 0$. 
Lemma 4.6 Let $V$ be an algebraic variety of pure dimension $k$ in $\mathbb{C}^{n}, \gamma$ a real simple curve in $\mathbb{C}^{n}$, and $d \leq 1$. Assume that $V$ is $(\gamma, d)$-hyperbolic at $\xi \in T_{\gamma, d} V \cap \mathbb{R}^{n}$ and let $\gamma_{\xi}(t):=\gamma(t)+\xi t^{d}$. Then there exist a convex zero neighborhood $G$ in $\mathbb{C}^{n}$ and $r>0$ such that $V$ satisfies $\operatorname{PL}\left(V, \Gamma\left(\gamma_{\xi}, d, G, \frac{1}{2} \bar{G}, r\right)\right)$. In particular, $V$ satisfies $\operatorname{PL}\left(V, \Gamma\left(\gamma_{\xi}, d, G, r\right)\right)$.

Lemma 4.7 Let $V$ be an algebraic variety of pure dimension $k$ in $\mathbb{C}^{n}, \gamma$ a real simple curve, $d \leq 1$, and $D$ a bounded open zero neighborhood in $\mathbb{C}^{n}$. Then the following assertions hold:

(a) If $V$ satisfies $\operatorname{PL}(V, \Gamma(\gamma, d, D, r))$ then for each zero neighborhood $G \subset D$ and $s \geq r$ the variety $V$ satisfies $\operatorname{PL}(V, \Gamma(\gamma, d, G, s))$.

(b) If for each $\xi \in D \cap T_{\gamma, d} V \cap \mathbb{R}^{n}$ there exist an open zero neighborhood $D_{\xi}$ in $\mathbb{C}^{n}$ and $r_{\xi}>1$ such that for $\gamma_{\xi}(t):=\gamma(t)+\xi t^{d}$, the variety $V$ satisfies $\operatorname{PL}\left(V, \Gamma\left(\gamma_{\xi}, d, D_{\xi}, r_{\xi}\right)\right)$ then for each compact set $K \subset D, V$ satisfies $\operatorname{PL}(V, \Gamma(\gamma, d, D, K, R))$ for each $R>1$.

In the following lemma, $\Gamma^{\prime}$ denotes a cone in $\mathbb{C}^{2}$. If $\pi: \mathbb{C}^{n} \rightarrow\{0\} \times \mathbb{C}^{2}$ is a projection we denote the corresponding surjection by $\tilde{\pi}: \mathbb{C}^{n} \rightarrow \mathbb{C}^{2}$, i.e., $\pi(z)=(0, \tilde{\pi}(z))$.

Lemma 4.8 Let $d \leq 1$ and let $\gamma$ be a real simple curve whose limit vector at infinity is $(0, \ldots, 0,1)$. Assume further that $0 \in T_{\gamma, d} V$ and that $\pi: \mathbb{C}^{n} \rightarrow \mathbb{C}^{n}$, $\pi\left(z^{\prime}, z_{n-1}, z_{n}\right):=\left(0, z_{n-1}, z_{n}\right)$ is noncharacteristic for $T_{\gamma, d} V$ at 0 . For each neighborhood $D$ of zero in $\mathbb{C}^{n}$ there are $\varepsilon_{1}, \varepsilon_{2}>0$ and $R_{1}>1$ such that $U_{1}:=$ $B^{n-2}\left(0, \varepsilon_{1}\right) \times B\left(0, \varepsilon_{2}\right)^{2}$ is a subset of $D$ and

$$
\pi: V \cap \Gamma\left(\gamma, d, U_{1}, R_{1}\right) \rightarrow \Gamma^{\prime}\left(\tilde{\pi} \circ \gamma, d, B\left(0, \varepsilon_{2}\right)^{2}, R_{1}\right)
$$

is proper.

Proof Fix a neighborhood $D$ of 0 in $\mathbb{C}^{n}$. There are $\varepsilon_{1}, \varepsilon_{2}>0$ such that

$$
B^{n-2}\left(0,2 \varepsilon_{1}\right) \times B\left(0,2 \varepsilon_{2}\right)^{2} \subset D
$$

and

$$
T_{\gamma, d} V \cap\left(\left(B^{n-2}\left(0,2 \varepsilon_{1}\right) \backslash \overline{B^{n-2}\left(0, \varepsilon_{1} / 2\right)}\right) \times B\left(0,2 \varepsilon_{2}\right)^{2}\right)=\emptyset .
$$

We have to show that there is $R_{1}$ such that for no $y$ in $\Gamma^{\prime}\left(\tilde{\pi} \circ \gamma, d, B\left(0, \varepsilon_{2}\right)^{2}, R_{1}\right)$ the fiber $V \cap \pi^{-1}(y)$ intersects the boundary of $\Gamma\left(\gamma, d, U_{1}, 0\right)$. Precise descriptions of the boundary of conoids are difficult. However, it is easy to see that

$$
\begin{aligned}
& \partial \Gamma\left(\gamma, d, U_{1}, 0\right) \cap \Gamma\left(\gamma, d, B^{n-2}\left(2 \varepsilon_{1}\right) \times B\left(0, \varepsilon_{2}\right)^{2}, R_{1}\right) \\
& \subset \Gamma\left(\gamma, d, \partial B^{n-2}\left(0, \varepsilon_{1}\right) \times B\left(0, \varepsilon_{2}\right)^{2}, 0\right) .
\end{aligned}
$$

We claim that there is $R_{1}$ such that the claim holds for $\varepsilon_{1}$ and $\varepsilon_{2}$ as chosen above. Assume this were wrong. In view of (4.1) this means that there is a sequence $\left(z_{j}\right)_{j \in \mathbb{N}}$ with $\lim _{j \rightarrow \infty}\left|z_{j}\right|=\infty$ and

$z_{j} \in V \cap \pi^{-1}\left(\Gamma^{\prime}\left(\tilde{\pi} \circ \gamma, d, B\left(0, \varepsilon_{2}\right)^{2}, 0\right)\right) \cap \Gamma\left(\gamma, d, \partial B^{n-2}\left(0, \varepsilon_{1}\right) \times B\left(0,2 \varepsilon_{2}\right)^{2}, 0\right)$ 
for all $j \in \mathbb{N}$. Each $z_{j}$ is of the form

$$
z_{j}=\gamma\left(t_{j}\right)+t_{j}^{d}\left(w_{j}^{\prime \prime}, w_{j, n-1}, w_{j, n}\right)
$$

with $\left|w_{j}^{\prime \prime}\right|=\varepsilon_{1},\left|w_{j, n-1}\right|<\varepsilon_{2}$, and $\left|w_{j, n}\right|<\varepsilon_{2}$. Furthermore, $\left(w_{j}^{\prime \prime}, w_{j, n-1}, w_{j, n}\right)$ is in $V_{\gamma, d, t_{j}}$ and $\lim _{j \rightarrow \infty} t_{j}=\infty$. A compactness argument implies that the sequence $\left(w_{j}^{\prime \prime}, w_{j, n-1}, w_{j, n}\right)_{j \in \mathbb{N}}$ has an accumulation point $w:=\left(w^{\prime \prime}, w_{n-1}, w_{n}\right)$. Then $w \in T_{\gamma, d} V$ with $\left|w^{\prime \prime}\right|=\varepsilon_{1},\left|w_{n-1}\right| \leq \varepsilon_{2}$, and $\left|w_{n}\right| \leq \varepsilon_{2}$. As this contradicts the choice of $\varepsilon_{2}$ and $\varepsilon_{1}$, the claim is proved.

Lemma 4.9 Let $\gamma, d, \pi$, and $D$ be as in Lemma 4.8. Assume, furthermore, that 0 is a singularity of $T_{\gamma, d} V$ which is not terminating for $\gamma$ and $d$, set $\delta:=\Delta(\gamma, d, 0)$, and fix a bounded set $K \subset \mathbb{C}^{n}$. Then $\varepsilon_{1}, \varepsilon_{2}$, and $R_{1}$ in Lemma 4.8 can be chosen in such a way that there are $\sigma, S>0$ such that with $U_{2}:=B^{n-2}(0, S) \times B(0, \sigma)^{2}$ we have $K \subset U_{2}$ and

$$
V \cap \Gamma\left(\gamma, d, U_{1}, R_{1}\right) \cap \pi^{-1}\left(\Gamma^{\prime}\left(\tilde{\pi} \circ \gamma, \delta, B(0, \sigma)^{2}, R_{1}\right)\right) \backslash \Gamma\left(\gamma, \delta, U_{2}, R_{1}\right)=\emptyset .
$$

Proof We assume that $U_{1}$ is so small that the origin is the only point in $V \cap U_{1}$ satisfying $\pi(z)=0$, and we fix $\varepsilon_{2}$ and $\varepsilon_{1}$ as in Lemma 4.8. In the sequel, the curve selection lemma from real algebraic geometry will be used. Hence we assume for the remainder of the proof that a semi-algebraic norm has been chosen on $\mathbb{C}^{n-2}$. This is no restriction since the claim is independent of the choice of the normonly the constants may vary. Since the claim is unchanged if we cut off all terms of $\gamma$ whose exponents are strictly smaller than $\delta$, we may also assume that $\gamma$ is a semi-algebraic function.

Fix $\sigma$ so large that $K \subset \mathbb{C}^{n-2} \times B(0, \sigma)^{2}$ and assume the claim were false for this value of $\sigma$ and arbitrarily large $S$. Then there is a sequence $\left(z_{j}\right)_{j \in \mathbb{N}}$ such that

$$
\begin{aligned}
z_{j} & \in V \cap \Gamma\left(\gamma, d, U_{1}, 0\right), & & j \in \mathbb{N}, \\
\tilde{\pi}\left(z_{j}\right) & \in \Gamma^{\prime}\left(\tilde{\pi} \circ \gamma, \delta, B(0, \sigma)^{2}, 0\right), & & j \in \mathbb{N}, \\
z_{j} & \notin \Gamma\left(\gamma, \delta, B^{n-2}(0, j) \times B(0, \sigma)^{2}, 0\right), & & j \in \mathbb{N}, \\
\lim _{j \rightarrow \infty}\left|z_{j}\right| & =\infty . & &
\end{aligned}
$$

Define

$$
\begin{aligned}
\Phi:] 0, \infty\left[\times U_{1} \backslash \mathbb{R} \times\{0\} \times \mathbb{C}^{2}\right. & \rightarrow \Gamma\left(\gamma, d, U_{1}, 0\right), \\
\left(t, w^{\prime \prime}, w_{n-1}, w_{n}\right) & \mapsto \gamma(t)+t^{d}\left(w^{\prime \prime}, w_{n-1}, w_{n}\right),
\end{aligned}
$$

and

$$
\begin{aligned}
\Psi:] 0, \infty\left[\times U_{1} \backslash \mathbb{R} \times\{0\} \times \mathbb{C}^{2}\right. & \rightarrow] 0, \infty\left[\times U_{1},\right. \\
\left(t, w^{\prime \prime}, w_{n-1}, w_{n}\right) & \mapsto\left(t^{\delta-d}\left|w^{\prime \prime}\right|^{-1}, w^{\prime \prime}, w_{n-1}, w_{n}\right),
\end{aligned}
$$

and let $M:=\Phi^{-1}\left(V \cap \Gamma^{\prime}\left(\tilde{\pi} \circ \gamma, \delta, B(0, \sigma)^{2}, 0\right)\right)$.

Note that $d$ and $\delta$ are rational since they are critical values. Hence $M$ is a semi-algebraic set and $\Phi$ and $\Psi$ are semi-algebraic functions. For $j \in \mathbb{N}$ pick $\tilde{w}_{j}$ 
with $\Phi\left(t_{j}, \tilde{w}_{j}\right)=z_{j}$. By (4.3), the first component of $\Psi\left(t_{j}, \tilde{w}_{j}\right)$ converges to zero. By (4.2) we have $\lim _{j \rightarrow \infty} \pi\left(\tilde{w}_{j}\right)=0$. Finally, a compactness argument shows that the sequence $\left(\tilde{w}_{j}-\pi\left(\tilde{w}_{j}\right)\right)_{j \in \mathbb{N}}$ admits an accumulation point. Hence there is a point of the form $\left(0, \zeta^{\prime \prime}, 0\right)$ in the boundary of $\Psi(M)$. By the curve selection lemma (see Bochnak, Coste, and Roy [1], Théorème 2.2.5), there is a semi-algebraic curve $L_{1}$ in $\Psi(M)$ leading to $\left(0, \zeta^{\prime \prime}, 0\right)$. Set $L:=\Psi^{-1}\left(L_{1}\right)$. Then $L$ admits a parametrization $\left(t, w^{\prime \prime}(t), w_{n-1}(t), w_{n}(t)\right)$ for large $t$ such that $\lim _{t \rightarrow \infty}\left(w^{\prime \prime}(t), w_{n-1}(t), w_{n}(t)\right)=\left(\zeta^{\prime \prime}, 0\right)$. Since this parametrization is semialgebraic, there are $A \neq 0$ and $a<0$ such that $t^{\delta-d}\left|w^{\prime \prime}(t)\right|^{-1}=t^{a}(A+o(1))$ as $t \rightarrow \infty$. This implies

$$
\left|w^{\prime \prime}(t)\right|=t^{\delta-d-a}\left(\frac{1}{A}+o(1)\right) \quad \text { as } t \rightarrow \infty .
$$

Since $\left|w^{\prime \prime}(t)\right|<\varepsilon_{1}$ by the choice of $U_{1}$, we have

$$
a>\delta-d \quad \text { or } \quad\left(a=\delta-d \text { and } \frac{1}{A} \leq \varepsilon_{1}\right) .
$$

Note that $\gamma(t)+t^{\delta-a} t^{d+a-\delta} w(t)=\gamma(t)+t^{d} w(t) \in V$. Hence $t^{d+a-\delta} w(t) \in$ $V_{\gamma, t, \delta-a}$. Since $\tilde{\pi}(w(t)) \in \Gamma^{\prime}\left(\tilde{\pi} \circ \gamma, \delta, B(0, \sigma)^{2}, 0\right)$ we have $\lim _{t \rightarrow \infty} w_{n-1}(t)=$ $\lim _{t \rightarrow \infty} w_{n}(t)=0$. Furthermore, the choices of $a$ and $A$ imply

$$
\lim _{t \rightarrow \infty} t^{d+a-\delta}\left|w^{\prime \prime}(t)\right|=1 / A
$$

Hence $T_{\gamma, \delta-a} V$ contains an element $\left(w^{\prime \prime}, 0,0\right)$ with $\left|w^{\prime \prime}\right|=1 / A$. We have to consider two cases:

If $\delta<\delta-a<d$, then $\delta-a$ is not a critical value. Hence $T_{\gamma, \delta-a} V$, being homogeneous by [8], Proposition 5 , contains the line through $\left(w^{\prime \prime}, 0,0\right)$ and the origin. This is a contradiction since the projection $\pi$ is noncharacteristic for $T_{\gamma, d} V$ at the origin and hence, by [9], Corollary 4.15, for $T_{\gamma, \delta-a} V$ at infinity.

If $a=\delta-d$, then the only element of the form $\left(w^{\prime \prime}, 0,0\right)$ in $T_{\gamma, d} V$ satisfying $\left|w^{\prime \prime}\right|<1 / A \leq \varepsilon_{1}$ is the origin. So we have a contradiction in this case also, and the proof is complete.

Lemma 4.10 Let $V$ be an algebraic surface in $\mathbb{C}^{n}$, let $\gamma$ be a real simple curve in standard parametrization, and let $d \leq 1$ be a critical value for $\gamma$ and $V$. Assume that $0 \in\left(T_{\gamma, d} V\right)_{\text {sing }}$ is not terminating for $\gamma$ and $d$ and set $\delta:=\Delta(\gamma, d, 0)$. Assume further that $\left.\pi: \mathbb{C}^{n} \rightarrow \mathbb{C}^{n}, \pi\left(z^{\prime}, z_{n-1}, z_{n}\right):=\left(0^{\prime}, z_{n-1}, z_{n}\right)\right)$ is noncharacteristic for $T_{\gamma, d} V$ at 0 and that $\left(0^{\prime}, 0,1\right)$ is the limit vector of $\gamma$ at infinity. If $V$ is $(\gamma, d, \delta)$-hyperbolic at 0 with respect to $\pi$, then there exist $\varepsilon_{1}, \varepsilon_{2}>0$, $S, \sigma, R \geq 1, R_{0} \geq R, 0<\eta_{1}<\varepsilon_{1}, 0<\eta_{2}<\varepsilon_{2}$, and $A_{0}, B \geq 1$ such that for $U:=B^{\bar{n}-2}\left(0, \varepsilon_{1}\right) \times B\left(0, \varepsilon_{2}\right)^{2}$ and $G:=B^{n-2}(0, S) \times B(0, \bar{\sigma})^{2}$ the following holds:

Whenever $u \in \operatorname{PSH}(V \cap \Gamma(\gamma, d, U, R))$ satisfies

$$
\begin{aligned}
& u(z) \leq|z|^{d} \text { for } z \in V \cap \Gamma(\gamma, d, U, R), \\
& u(z) \leq 0 \text { for } z \in V \cap \mathbb{R}^{n} \cap \Gamma(\gamma, d, U, R),
\end{aligned}
$$


then

$$
\begin{aligned}
& u(z) \leq A_{0}|\operatorname{Im} z| \\
& \quad \text { for } z \in V \cap \Gamma\left(\gamma, d, B^{n-2}\left(0, \eta_{1}\right) \times B\left(0, \eta_{2}\right)^{2}, R_{0}\right) \backslash \Gamma(\gamma, \delta, G, 0)
\end{aligned}
$$

and

$$
u(z) \leq B|z|^{\delta} \text { for } z \in V \cap \Gamma\left(\gamma, \delta, 4 G, R_{0}\right) .
$$

Proof It is clear that the claim is independent of the choice of the norm, only the constants may vary. Therefore, we use the maximum norm in this proof.

Let $R$ be as in the Definition 3.13 of $(\gamma, d, \delta)$-hyperbolicity and let $D$ and $K$ be the neighborhoods of zero which are denoted by $U$ and $G$ in that definition, i.e., if $z \in V \cap \Gamma(\gamma, d, D, R)$ satisfies $\pi(z) \in \mathbb{R}^{2}$ and $\pi(z) \notin \Gamma^{\prime}(\pi \circ \gamma, \delta, \pi(K), 0)$, then $z$ is real. It is not difficult to see the existence of $C_{1}$ such that $\pi\left(\gamma(t)+t^{\delta} w\right) \notin$ $\Gamma^{\prime}(\pi \circ \gamma, \delta, \pi(K), 0)$ whenever $w \in \mathbb{C}^{n}$ satisfies $\left|w_{n-1}\right| \geq C_{1}$.

We apply Lemma 4.9 to obtain $\varepsilon_{1}, \varepsilon_{2}, R_{1}, \sigma, S, U_{1}$, and $U_{2}$ as in that lemma such that $U_{1} \subset D$ and $K \subset U_{2}$. Define $U$ and $G$ as in the hypothesis. Set $\eta_{1}:=$ $\min \left(1 / 4, \varepsilon_{1} / 2\right)$ and $\eta_{2}:=\min \left(1 / 4, \varepsilon_{2} / 2\right)$. Choose $C_{2}$ such that $|z| \leq C_{2}|\pi(z)|$ whenever $z \in \Gamma\left(\gamma, d, U, R_{1}\right)$. Let $R_{0} \geq \max \left(R, R_{1}\right)$ be sufficiently large.

Let $u \in \operatorname{PSH}(V \cap \Gamma(\gamma, d, U, R))$ satisfying (4.5) be given, and fix $z \in V \cap$ $\Gamma\left(\gamma, d, B^{n-2}\left(0, \eta_{1}\right) \times B^{2}\left(0, \eta_{2}\right), R_{0}\right)$. Then there are

$$
t^{\prime}>R_{0} \text { and } w^{\prime} \in B^{n-1}\left(0, \eta_{1}\right) \times B^{2}\left(0, \eta_{2}\right)
$$

such that $z=\gamma\left(t^{\prime}\right)+\left(t^{\prime}\right)^{d} w^{\prime}$. We set $t:=t^{\prime}+\left(t^{\prime}\right)^{d} \operatorname{Re} w_{n}^{\prime}$ and define

$$
w:=t^{-d}\left(\gamma\left(t^{\prime}\right)-\gamma(t)\right)+\left(t^{\prime} / t\right)^{d} w^{\prime} .
$$

This implies

$$
z=\gamma(t)+t^{d} w
$$

Since $\left|w^{\prime}\right|<\eta_{2} \leq 1 / 4$, the mean value theorem shows that $w \in B^{n-2}\left(0,2 \eta_{1}\right) \times$ $B^{2}\left(0,2 \eta_{2}\right)$ provided $R_{0}$ is sufficiently large. The advantage of $w$ over $w^{\prime}$ is that $\operatorname{Re} w_{n}=0$ since $\gamma$ is in standard parametrization. These values of $t$ and $w$ will be fixed for the remainder of the proof.

Define

$$
a: B^{2}(0,1) \rightarrow \mathbb{C}^{2}, \quad a(\zeta):=\pi \circ \gamma(t)+t^{d} \varepsilon_{2} \zeta
$$

Then $a(\zeta) \in \Gamma^{\prime}\left(\pi \circ \gamma, d, B^{2}\left(0, \varepsilon_{2}\right), R_{0} / 2\right)$ for all $\zeta \in B^{2}(0,1)$. Furthermore, we have $|a(\zeta)|<2 t+\varepsilon_{2} t^{d}<3 t$ for all $\zeta \in B^{2}(0,1)$ provided $R_{0}$ is chosen sufficiently large. Similarly, we have $|a(\zeta)|>t / 3$ for all $\zeta \in B^{2}(0,1)$.

Set $C_{3}:=\max \left(3^{d} C_{2}^{d}, 3^{-d}\right)$ and define

$$
\begin{aligned}
v: B^{2}(0,1) & \rightarrow[-\infty, \infty[ \\
v(\zeta) & :=C_{3}^{-1} t^{-d} \max \left\{u(z): z \in V \cap\left(\gamma(t)+t^{d} U\right), \pi(z)=a(\zeta)\right\} .
\end{aligned}
$$


We claim that $v$ is plurisubharmonic. For those points $\zeta$ for which all points in $\pi^{-1}(\zeta) \cap V \cap\left(\gamma(t)+t^{d} U\right)$ are regular points of $V$ this is clear since the restriction of $\pi$ to $V \cap \Gamma\left(\gamma, d, U, R_{0} / 2\right)$ is proper by Lemma 4.8. In the other points, the argument in Hörmander [12], Lemma 4.4, can be used to show that the singularity of $v$ is removable.

For $\zeta \in B^{2}(0,1)$ fix $y \in V \cap\left(\gamma(t)+t^{d} U\right)$ with $\pi(y)=a(\zeta)$. Then $|y| \leq$ $C_{2}|a(\zeta)| \leq 3 C_{2} t$ and $|y| \geq|a(\zeta)| \geq t / 3$. This implies in particular that $|y|^{d} \leq$ $C_{3} t^{d}$. Hence the first inequality of (4.5) implies $v(\zeta) \leq 1$.

Next assume that $R_{0}$ is so large that $\varepsilon_{3}:=C_{1} \varepsilon_{2}^{-1} t^{\delta-d}$ satisfies $\varepsilon_{3}<1 / 2$ and consider $\zeta \in B^{2}(0,1) \cap \mathbb{R}^{2}$ with $\left|\zeta_{1}\right| \geq \varepsilon_{3}$. Fix $y \in V \cap\left(\gamma(t)+t^{d} U\right)$ with $\pi(y)=a(\zeta)$. There is $x \in U$ with $y=\gamma(t)+t^{d} x$. Then

$$
\left|x_{n-1}\right|=t^{d} \varepsilon_{2}\left|\zeta_{1}\right| \geq t^{d} \varepsilon_{2} \varepsilon_{3}=C_{1} t^{\delta} .
$$

In particular, $\pi(y) \notin \Gamma^{\prime}(\pi \circ \gamma, \delta, \pi(K), 0)$. Hence $y$ is real by the choice of $K$. Since this holds for all such $y$, we have $v(\zeta) \leq 0$ by the second inequality of (4.5).

Set $\lambda:=\sigma C_{1}^{-1}$. We have verified the hypotheses of [3], Lemma 5.8, with $\varepsilon_{3}$ in the place of $\varepsilon$. This lemma-which is basically an estimate for the harmonic measure of the slit polydisk - together with [3], Lemma 5.7, implies the existence of a constant $C_{4}$, depending only on $\lambda$, such that

$$
v(\zeta) \leq \begin{cases}C_{4}\left(\left|\operatorname{Im} \zeta_{1}\right|+\left|\operatorname{Im} \zeta_{2}\right|\right), & \lambda \varepsilon_{3} \leq\left|\zeta_{1}\right|<1 / 2,\left|\zeta_{2}\right|<1 / 2, \\ C_{4}\left(\varepsilon_{3}+\left|\operatorname{Im} \zeta_{2}\right|\right), & \left|\zeta_{1}\right|<\lambda \varepsilon_{3},\left|\zeta_{2}\right|<1 / 2 .\end{cases}
$$

We have to evaluate (4.9) at the point $z$ which was chosen initially. To do so, fix $\zeta \in B^{2}(0,1)$ such that $a(\zeta)=\pi(z)$. Then $\varepsilon_{2} \zeta=\left(w_{n-1}, w_{n}\right)$. In particular, $\left|\zeta_{1}\right|,\left|\zeta_{2}\right|<1 / 2$. We start with the proof of (4.6). This inequality follows immediately from the first line of (4.9) if $\left|\zeta_{1}\right| \geq \lambda \varepsilon_{3}$. So assume $\left|\zeta_{1}\right|<\lambda \varepsilon_{3}$. We only have to prove (4.6) if $z \notin \Gamma(\gamma, \delta, G, 0)$. Then Lemma 4.9 implies $\pi(z) \notin$ $\Gamma^{\prime}(\pi \circ \gamma, \delta, \pi(G), 0)$. Hence $t^{d-\delta}|\pi(w)| \geq \sigma$ and $|\zeta| \geq \varepsilon_{2}^{-1} \sigma t^{\delta-d}=\lambda \varepsilon_{3}$. Since $\left|\zeta_{1}\right|<\lambda \varepsilon_{3}$, this implies $\left|\zeta_{2}\right| \geq \lambda \varepsilon_{3}$. However, $\zeta_{2}$ is purely imaginary by the choice of $w$. Hence the first estimate of (4.9) implies

$$
v(\zeta) \leq C_{4}\left(\varepsilon_{3}+\left|\operatorname{Im} \zeta_{2}\right|\right) \leq C_{4}\left(\frac{1}{\lambda}\left|\operatorname{Im} \zeta_{2}\right|+\left|\operatorname{Im} \zeta_{2}\right|\right) .
$$

This completes the proof of (4.6).

Now we prove (4.7). We only have to do this if $z \in \Gamma(\gamma, \delta, 4 G, 0)$. In this case, $t^{\delta-d}|\pi(w)|<4 \sigma$ and hence $|\zeta|<4 \lambda \varepsilon_{3}$. If the first line of (4.9) is applicable, we get $v(\zeta) \leq 8 C_{4} \lambda \varepsilon_{3}$, otherwise $v(\zeta) \leq C_{4}(1+4 \lambda) \varepsilon_{3}$. In both cases, (4.7) is shown.

Definition 4.11 A pure dimensional algebraic variety $V$ in $\mathbb{C}^{n}$ is said to satisfy the strong radial Phragmén-Lindelöf condition (SRPL) if there are constants $A \geq 1$, $B \geq 0$ such that each $u \in \operatorname{PSH}(V)$ which satisfies

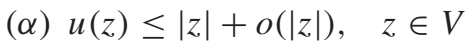

( $\beta) ~ u(z) \leq 0, \quad z \in V \cap \mathbb{R}^{n}$

also satisfies 
$(\gamma) u(z) \leq A|z|+B, \quad z \in V$.

Proof of Theorem $4.3(a) \Rightarrow$ (b): This holds by Theorem 3.16.

(b) $\Rightarrow$ (c): This holds by the arguments that we gave in the proof of Corollary 4.4 to derive condition (1") from condition 3.15 (1).

(c) $\Rightarrow$ (a): Since $V_{h}$ is homogeneous and satisfies (SPL) by hypothesis, each irreducible component $W$ of $V_{h}$ satisfies $\mathrm{PL}_{\mathrm{loc}}(0)$. Hence $W$ satisfies the dimension condition by the Remark after 3.6. In particular, each irreducible component $W$ of $V_{h}$ has real regular points. By condition (3) of 4.3 (c) $V$ is $\left(\gamma_{\xi}, 1\right)$-hyperbolic at such a point $\xi \in W \cap S^{n-1}$ for $\gamma_{\xi}(t):=t \xi$. In the notation of [4], 2.8, this means that $V$ is locally hyperbolic at infinity in the direction $\xi$. Hence $V$ satisfies the condition (SRPL) by [4], Theorem 5.1. By condition (1) of 4.3 (c), $V$ satisfies $\mathrm{PL}_{\text {loc }}(\xi)$ at each $\xi \in V \cap \mathbb{R}^{n}$. Therefore, two of the three conditions in Meise and Taylor [14], Proposition 4.5, are satisfied and it follows that $V$ satisfies (SPL) if the following condition holds:

For each $\xi \in V_{h} \cap S^{n-1}$ there exist $A \geq 1, R \geq 1$, and $0<\delta<1$ such that for $\gamma_{\xi}(t):=t \xi, \quad t \geq 1$, each $u \in \operatorname{PSH}(V)$ which satisfies the conditions $(\alpha)$ and $(\beta)$ of 3.1 also satisfies $u(z) \leq$ $A|\operatorname{Im} z|, z \in V \cap \Gamma(\gamma, 1, B(0, \delta), R)$.

To prove that (4.10) holds, recall from 4.2 that there we defined

$$
M_{0}:=\left\{\xi \in\left(V_{h}\right)_{\operatorname{sing}} \cap S^{n-1}: \xi \text { is not terminating }\right\}
$$

and that $M_{0}=\emptyset$ is equivalent to $\mathcal{K}=\emptyset$. Then note that by the present hypothesis, $V$ is $\left(\gamma_{\xi}, 1\right)$-hyperbolic at $0 \in T_{\gamma_{\xi}, 1} V$ for each $\xi \in V_{h} \cap S^{n-1} \backslash M_{0}$ and $\gamma_{\xi}(t):=t \xi$. Hence Lemma 4.6 implies that condition (4.10) holds for each $\xi \in V_{h} \cap S^{n-1} \backslash M_{0}$. Therefore, $V$ satisfies (SPL) whenever $M_{0}=\emptyset$ or equivalently $\mathcal{K}=\emptyset$.

To complete the proof if $\mathcal{K} \neq \emptyset$ we define for each $\xi \in M_{0}$ the sets

$$
\begin{aligned}
& \mathcal{R}_{\xi}:=\left\{C \in \mathcal{R}(V): C=\left(\zeta_{j}, d_{j}\right)_{j=1}^{l}, \zeta_{1}=\xi\right\}, \\
& \mathcal{K}_{\xi}:=\left\{\left(\gamma_{C}, d_{l-1}, d_{l}\right): C \in \mathcal{R}_{\xi}\right\} .
\end{aligned}
$$

By the definition of $\mathcal{R}(V)$ there exists $D \in \mathcal{R}_{\xi}$ with $D=\left(\zeta_{j}, d_{j}\right)_{j=1}^{2}$. Since $\gamma_{D}(t)=\zeta_{1} t=\xi t=\gamma_{\xi}(t)$, and since $d_{1}=1$, the triple $\left(\gamma_{\xi}, 1, d_{2}\right)$ belongs to $\mathcal{K}_{\xi}$. Now we claim that the following assertion holds:

For each $\xi \in M_{0}$, and each $(\gamma, d, \delta) \in \mathcal{K}_{\xi}$ there exist a zero neighborhood $G$ in $\mathbb{C}^{n}$ and $r>0$ such that $V$ satisfies $\operatorname{PL}(V, \Gamma(\gamma, d, G, r))$.

By the preceding considerations, (4.11) implies that (4.10) holds for all $\xi \in M_{0}$ and hence for all $\xi \in V_{h} \cap S^{n-1}$. Thus, the proof is complete once the claim (4.11) is proved.

To prove (4.11) we fix $\xi \in M_{0}$ and note that $\mathcal{R}_{\xi}$ has a natural order structure, which is defined in the following way: For $C, D \in \mathcal{R}_{\xi}$ let $C \leq D$ if $l(C) \leq l(D)$ and if the first $l(C)$ components of $C$ and $D$ coincide. Obviously, this ordering induces an order structure of $\mathcal{K}$. It can be used to prove (4.11) in the following 
way: In step 1 we show that (4.11) holds for each maximal element $(\gamma, d, \delta)$ of $\mathcal{K}_{\xi}$. Then we show in step 2 that (4.11) holds for $(\gamma, d, \delta) \in \mathcal{K}_{\xi}$ which is not maximal, provided that (4.11) holds for all $(\kappa, s, \sigma) \in \mathcal{K}_{\xi}$ which are direct successors of $(\gamma, d, \delta)$. By an obvious induction over the length of $C \in \mathcal{R}_{\xi}$ these two steps imply that (4.11) holds for each $\xi \in M_{0}$.

Step 1: Assume that $(\gamma, d, \delta)$ is a maximal element of $\mathcal{K}_{\xi}$. By the definition of a normal critical set this implies that all points of $T_{\gamma, \delta} V \cap \mathbb{R}^{n}$ are either regular points or terminating singularities for $\gamma$ and $\delta$. To prove that $V$ satisfies $\operatorname{PL}(V, \Gamma(\gamma, d, G, r))$ for some neighborhood $G$ in $\mathbb{C}^{n}$ and some $r>1$, note first that by hypothesis, $V$ is $(\gamma, d, \delta)$-hyperbolic at $0 \in T_{\gamma, d} V$ and that for each $\zeta \in T_{\gamma, \delta} V \cap \mathbb{R}^{n}$ the variety $V$ is $(\gamma, \delta)$-hyperbolic at $\zeta$. Hence we can apply Lemma 4.10. Using the notation which was introduced in this lemma, let $\Gamma:=\Gamma(\gamma, d, U, R)$ and fix $u \in \operatorname{PSH}(V \cap \Gamma)$ satisfying condition (4.5). Then Lemma 4.10 implies that for $U_{0}:=B^{n-2}\left(0, \eta_{1}\right) \times B\left(0, \eta_{2}\right)^{2}$ we have the estimates

$$
u(z) \leq A_{0}|\operatorname{Im} z|, z \in V \cap \Gamma\left(\gamma, d, U_{0}, R_{0}\right) \backslash \Gamma\left(\gamma, \delta, G, R_{0}\right)
$$

and

$$
u(z) \leq B|z|^{\delta}, z \in V \cap \Gamma\left(\gamma, \delta, 4 G, R_{0}\right) .
$$

Since we remarked already that $V$ is $\left(\gamma_{\zeta}, \delta\right)$-hyperbolic at each $\zeta \in T_{\gamma, \delta} V \cap \mathbb{R}^{n}$, it follows from Lemma 4.6, that there exist a zero neighborhood $G_{\zeta}$ in $\mathbb{C}^{n}$ and $R_{\zeta}>1$ such that $V$ satisfies $\operatorname{PL}\left(V, \Gamma\left(\gamma_{\zeta}, \delta, G_{\zeta}, R_{\zeta}\right)\right)$ or equivalently $\operatorname{PL}(V, \Gamma(\gamma, \delta, \zeta+$ $\left.\left.G_{\zeta}, R_{\zeta}\right)\right)$ for each $\zeta \in\left(T_{\gamma, \delta} V\right) \cap \mathbb{R}^{n}$. By Lemma $4.7(\mathrm{~b})$, this implies that $V$ satisfies $\operatorname{PL}\left(V, \Gamma\left(\gamma, \delta, 4 G, 2 \bar{G}, R_{0}\right)\right)$. Hence it follows from the estimate (4.13) that there exists $A_{1}$, not depending on $u$, so that

$$
u(z) \leq A_{1}|\operatorname{Im} z|, z \in V \cap \Gamma\left(\gamma, \delta, 2 G, R_{0}\right) .
$$

From (4.14) and (4.12) it follows that $V$ satisfies $\operatorname{PL}\left(V, \Gamma\left(\gamma, d, U_{0}, R_{0}\right)\right)$. Hence we proved (4.11) in the case that $(\gamma, d, \delta)$ is a maximal element of $\mathcal{R}_{\xi}$.

Step 2: Assume that $(\gamma, d, \delta) \in \mathcal{K}_{\xi}$ is not maximal and that (4.11) holds for each $(\kappa, s, \sigma) \in \mathcal{K}_{\xi}$ which is a direct successor of $(\gamma, d, \delta)$. Then choose $C=$ $\left(\zeta_{j}, d_{j}\right)_{j=1}^{l}$ in $\mathcal{R}_{\xi}$ so that $(\gamma, d, \delta)=\left(\gamma_{C}, d_{l-1}, d_{l}\right)$. Since $(\gamma, d, \delta)$ is not maximal, there is $D \in \mathcal{R}_{\xi}$ with $D>C$. This implies that $T_{\gamma, \delta} V=T_{\gamma_{C}, d_{l}} V$ contains a real singular point which is not terminating for $\gamma$ and $\delta$. If $\zeta$ is such a point, define $\gamma_{\zeta}$ by $\gamma_{\zeta}(t):=\gamma(t)+\zeta t^{\delta}$ and let $\zeta_{l+1}:=\zeta$ and $d_{l+1}:=\Delta\left(\gamma_{\zeta}, \delta, \zeta\right)$. Then $C_{\zeta}:=\left(\zeta_{j}, d_{j}\right)_{j=1}^{l+1}$ is a normal critical set which is a direct successor of $C$. Hence the present hypothesis implies that $V$ satisfies $\operatorname{PL}\left(V, \Gamma\left(\gamma_{\zeta}, \delta, G_{\zeta}, R_{\zeta}\right)\right)$ for some $R_{\zeta}>1$ and zero neighborhood $G_{\zeta}$ in $\mathbb{C}^{n}$. At each $\zeta \in T_{\gamma, \delta} V \cap \mathbb{R}^{n}$ which is regular or terminating for $\gamma$ and $\delta$, the variety $V$ is $\left(\gamma_{\zeta}, \delta\right)$-hyperbolic by condition (4) of 4.3 (c). By Lemma 4.6, this implies that $V$ satisfies $\operatorname{PL}\left(V, \Gamma\left(\gamma_{\zeta}, \delta, G_{\zeta}, R_{\zeta}\right)\right)$ for some zero neighborhood $G_{\zeta}$ in $\mathbb{C}^{n}$ and some $R_{\zeta}>1$.

Next note that $V$ is $(\gamma, d, \delta)$-hyperbolic at $0 \in T_{\gamma, d} V$ by condition (4) of 4.3 (c). Hence we conclude from Lemma 4.10 as in the first step that each $u \in \operatorname{PSH}(V \cap \Gamma)$ which satisfies (4.5) also satisfies the estimates (4.12) and (4.13). By the considerations above, we conclude as before that $\operatorname{PL}\left(V, \Gamma\left(\gamma, \delta, 4 G, \overline{2 G}, R_{0}\right)\right)$ is satisfied for $V$. It follows as before that $V$ satisfies $\operatorname{PL}\left(V, \Gamma\left(\gamma, d, U_{0}, R_{0}\right)\right)$. Thus we showed that (4.11) holds for $(\gamma, d, \delta)$. As we already indicated, this completes the proof of Theorem 4.3. 
To complete the proof of Theorem 3.10, we need the following lemma, whose proof is essentially the same as that of [5], Lemma 3.19.

Lemma 4.12 Let $V$ be an algebraic variety of pure dimension $k$ in $\mathbb{C}^{n}$, let $\gamma$ be a real simple curve in $\mathbb{C}, d \leq 1$, and $\kappa \in\left(T_{\gamma, d} V\right)_{\mathrm{reg}} \cap \mathbb{R}^{n}$. If $V$ is $(\gamma, d)$-hyperbolic at $\kappa$ then $V$ is $(\gamma, d)$-hyperbolic at $\kappa$ with respect to each projection $\pi$ in $\mathbb{C}^{n}$ which is noncharacteristic for $T_{\gamma, d} V$ at $\kappa$.

Remark 4.13 To complete the proof of Theorem 3.10, we show that 3.10 (b) implies 3.10 (a) and that 3.10 (b) is equivalent to 3.10 (c). To prove the first implication we argue as follows: As in the proof of Theorem 4.3, the present hypothesis and Meise and Taylor [14], Proposition 4.5, imply that $V$ satisfies (SPL) if we show that condition (4.10) is fulfilled. Now note that for each $\eta \in V \cap S^{n-1}$ and $\gamma_{\eta}(t):=t \eta$, the variety $V$ is $\left(\gamma_{\eta}, 1\right)$-hyperbolic at 0 by the present hypothesis. Hence Lemma 4.6 implies condition (4.10).

To show that 3.10 (b) and 3.10 (c) are equivalent, note first that 3.10 (c) for some projection $\pi$ obviously implies 3.10 (b). For the converse implication let $\pi$ be a projection in $\mathbb{C}^{n}$ which is noncharacteristic for $V_{h}$ at infinity. Then the present hypotheses imply that $V_{h}$ is given by (3.1) with $a_{j} \in \mathbb{R}$ and $a_{j} \notin \operatorname{ker} \pi$, $1 \leq j \leq \mu$. Therefore, it is easy to check that 3.10 (b) together with Lemma 4.12 implies the assertions of 3.10 (c) for the projection $\pi$. Thus the proof of Theorem 3.10 is complete.

\section{Examples}

In this section we provide some examples which show how the main results of the present paper can be applied. We begin with two examples concerning algebraic curves in $\mathbb{C}^{2}$.

Example 5.1 Let $P \in \mathbb{C}[x, y]$ be defined as

$$
P(x, y):=x^{3}-x^{2} y-x y^{2}+y^{3}+x^{2}-y^{2}-1 .
$$

Then $V(P)$ satisfies (SPL), while the principal part $P_{3}$ of $P$ is not square-free.

To show that $V(P)$ satisfies (SPL), we check the conditions of Corollary 3.11. Note first that $\operatorname{grad} P$ does not vanish on $V(P)$. Since $P$ has real coefficients, it follows from this, the implicit function theorem, and [5], Proposition 3.16, that $V(P)$ satisfies $\operatorname{PL}_{\mathrm{loc}}(\xi)$ for each $\xi \in V(P) \cap \mathbb{R}^{2}$. Hence condition 3.11 (a) is satisfied.

To show that also condition (b) of 3.11 is fulfilled, note that for each $x \in \mathbb{R}$, $|x|>3$ the equation $P(x, y)=0$ has three different real solutions. To show this, note first that

$$
P(x, y)=(x+y)(x-y)(x-y+1)-1 .
$$

From this we get that for $x>3$ we have:

$$
P(x, y)<0 \text { if } y<-x \text { or } y=x \text {, while } P(x, y)>0 \text { if } y=0 \text { or } y>x+2 \text {. }
$$


For $x<-3$ we have

$$
\begin{aligned}
& P(x, y)<0 \text { if } y<x \text { or } y=0, \text { while } \\
& \quad P(x, y)>0 \text { if } y=x+\frac{1}{2} \text { or } y>2-x .
\end{aligned}
$$

Obviously, these sign changes imply that condition (b) of 3.11 holds for $\xi=(1,0)$. Hence $V(P)$ satisfies (SPL) by Corollary 3.11. However,

$$
P_{3}(x, y)=(x-y)^{2}(x+y)
$$

is not square-free.

Example 5.2 Let $P \in \mathbb{C}[x, y]$ be defined by

$$
P(x, y):=4 x^{4}-5 y^{2} x^{2}+y^{4}-8 x^{2}-8 y^{2}+3 .
$$

Then $V(P)$ satisfies (SPL) and $V(P) \cap \mathbb{R}^{2}$ contains a connected component which is bounded. As in the previous example it can be derived from Corollary 3.11 that $V(P)$ satisfies (SPL). To show that $V(P) \cap \mathbb{R}^{2}$ has a bounded connected component, note first that $P(0,0)=3>0$. Since it is easy to check that $P$ is negative on the boundary of the rectangle $R:=[-1,1] \times[-2,2], V(P) \cap \mathbb{R}^{2}$ must have a bounded component in the interior of $R$.

To treat examples of surfaces in $\mathbb{C}^{3}$ in a convenient way we will use the following lemma. It can be proved by an obvious modification of the proof of [6], Lemma 5.1.

Lemma 5.3 Let $P \in \mathbb{R}\left[z_{1}, \ldots, z_{n}\right] \backslash \mathbb{C}$, a real simple curve $\gamma$ with Puiseux series expansion $\gamma(t)=\sum_{j=-\infty}^{q} a_{j} t^{j / q}$, and $\left.\left.d \in \mathbb{Q} \cap\right]-\infty, 1\right]$ be given. Expand

$$
P(\gamma(t)+w)=\sum_{j \in \mathbb{Z}, \alpha \in \mathbb{N}_{0}^{n}} a_{j, \alpha} t^{j / q} w^{\alpha}=\sum_{k=0}^{\infty} F_{\omega_{k}}(w, t),
$$

where $\omega_{0}=\omega_{0}(d):=\max \left\{j / q+d|\alpha|: a_{j, \alpha} \neq 0\right\}$ and

$$
F_{\omega_{k}}(w, t)=\sum_{j / q+d|\alpha|=\omega_{k}} a_{j, \alpha} t^{j / q} w^{\alpha} .
$$

If $\frac{\partial F_{\omega_{0}}}{\partial z_{n}}(\xi, 1) \neq 0$ for some $\xi \in V\left(F_{\omega_{0}}(\cdot, 1)\right) \cap \mathbb{R}^{n}$, then $V(P)$ is $(\gamma, d)$-hyperbolic at $\xi$ with respect to the projection $\pi: \mathbb{C}^{n} \rightarrow \mathbb{C}^{n}, \pi\left(z^{\prime}, z_{n}\right):=\left(z^{\prime}, 0\right)$.

Remark 5.4 For an algebraic surface $V$ in $\mathbb{C}^{n}$ one can determine the set $\mathcal{K}(V)$ of relevant triples for $V$ directly by induction. To do so let

$$
M_{0}:=\left\{\xi \in\left(V_{h}\right)_{\text {sing }} \cap S^{n-1}: \xi \text { is not terminating }\right\} .
$$

If $M_{0}=\varnothing$ then let $\mathcal{K}(V):=\varnothing$. Otherwise fix $\xi \in M_{0}$, define the curve $\gamma \xi$ by $\gamma_{\xi}(t):=t \xi, t \geq 1$, and let

$$
\mathcal{K}_{1}:=\left\{\left(\gamma_{\xi}, 1, \Delta\left(\gamma_{\xi}, 1,0\right)\right): \xi \in M_{0}\right\}
$$


If $\mathcal{K}_{j} \neq \varnothing$ is already constructed then let

$$
\begin{aligned}
\mathcal{K}_{j, c}:=\{(\gamma, d, \delta) & \in \mathcal{K}_{j}: \text { there is } \zeta \in\left(T_{\gamma, \delta} V\right)_{\text {sing }} \cap \mathbb{R}^{n} \\
& \text { which is not terminating for } \gamma \text { and } \delta\} .
\end{aligned}
$$

If $\mathcal{K}_{j, c}=\emptyset$ then define $\mathcal{K}(V):=\varnothing$. Otherwise fix $(\gamma, d, \delta) \in \mathcal{K}_{j, c}$, denote by $\eta$ the limit vector of $\gamma$ at infinity, and let $M_{(\gamma, d, \delta)}:=\left\{\zeta \in\left(T_{\gamma, \delta} V\right)_{\text {sing }} \cap \mathbb{R}^{n}:\langle\zeta, \eta\rangle=0, \zeta\right.$ not terminating for $\gamma$ and $\left.\delta\right\}$.

For $\zeta \in M_{(\gamma, d, \delta)}$ let $\gamma_{\zeta}(t):=\gamma(t)+\zeta t^{\delta}$ and define

$$
\mathcal{K}_{j+1}:=\left\{\left(\gamma_{\zeta}, \delta, \Delta(\gamma, \delta, \zeta)\right): \zeta \in M_{(\gamma, d, \delta)},(\gamma, d, \delta) \in \mathcal{K}_{j, c}\right\}
$$

Since it is easy to check that

$$
\mathcal{K}_{j}=\left\{\left(\gamma_{C}, d_{j}, d_{j+1}\right): C \in \mathcal{R}(V), l(C)=j+1\right\},
$$

the procedure ends after finitely many steps and gives $\mathcal{K}(V)=\bigcup_{j=1}^{N} \mathcal{K}_{j}$ for $N:=\max \{l(C)-1: C \in \mathcal{R}(V)\}$.

Example 5.5 If $P \in \mathbb{R}[x, y, z]$ is defined as

$$
P(x, y, z):=\frac{1}{2} y\left(x^{2}-y^{2}\right)-(x-y) z+z,
$$

then $V(P)$ satisfies (SPL).

To derive this from Theorem 4.3, note first that $\operatorname{grad} P$ does not vanish on $V(P)$. Hence each point $\xi \in V(P) \cap \mathbb{R}^{3}$ is a regular point and $V(P)$ satisfies $\operatorname{PL}_{\text {loc }}(\xi)$ by [5], Proposition 7.4. Thus condition (1) of Theorem 4.3 (c) is fulfilled. Next note that for $V=V(P)$ we have

$$
V_{h}=V(Q) \text { for } Q(x, y, z):=y\left(x^{2}-y^{2}\right) .
$$

Hence $V_{h}$ is the union of three complex planes, each of which has real generators. Consequently, $V_{h}$ satisfies (SPL). Whenever $\xi \in V_{h} \cap \mathbb{R}^{3}$ is a regular point of $V_{h}$ then $\operatorname{grad} Q(\xi) \neq 0$. Hence it follows from Lemma 5.3 that $V$ is $\left(\gamma_{\xi}, 1\right)$-hyperbolic at $\xi$ for $\gamma_{\xi}(t):=t \xi$. To show that each $\xi \in\left(V_{h}\right)_{\text {sing }} \cap S^{2}$ is not terminating, assume that there is such a point $\xi$ which is terminating. Then it follows from [9], Proposition 4.17, that there exists a simple curve $\sigma$ which satisfies $\operatorname{tr}(\sigma) \subset V_{\text {sing }}$. However, this is a contradiction, since we remarked already that all points of $V$ are regular. Hence the conditions (2) and (3) of 4.3 (c) are fulfilled.

To show that also condition (4) of 4.3 (c) holds, note that

$$
\left(V_{h}\right)_{\text {sing }} \cap S^{2}=\{(0,0,1),(0,0,-1)\}=: M_{0} .
$$

If we let $\gamma_{ \pm}(t):=(0,0, \pm t), t>0$, then a direct computation using [8], Corollary 4 , shows

$$
\begin{aligned}
T_{\gamma_{ \pm}, d} V & =V_{h}, 1 / 2<d \leq 1, \\
T_{\gamma_{ \pm}, 1 / 2} V & =\left\{(x, y, z) \in \mathbb{C}^{3}:(x-y)(y(x+y) / 2 \mp 1)=0\right\} .
\end{aligned}
$$


Thus we get from 4.2 that

$$
\mathcal{K}_{1}=\left\{\left(\gamma_{+}, 1, \frac{1}{2}\right),\left(\gamma_{-}, 1, \frac{1}{2}\right)\right\} .
$$

It is easy to check that $T_{\gamma_{-}, 1 / 2} V$ has no real singularities while

$$
\left(T_{\gamma_{+}, 1 / 2} V\right)_{\text {sing }} \cap \mathbb{R}^{3}=\{(1,1, z): z \in \mathbb{R}\} \cup\{(-1,-1, z): z \in \mathbb{R}\} .
$$

Hence we have $\mathcal{K}_{1, c}=\left\{\left(\gamma_{+}, 1,1 / 2\right)\right\}$. Note that by the same argument as before, the points $(1,1,0)$ and $(-1,-1,0)$ of $\left(T_{\gamma_{+}, 1 / 2} V\right)_{\text {sing }}$ are not terminating for $\gamma_{+}$ and $d=1 / 2$. If we define $\sigma_{+}(t):=(\sqrt{t}, \sqrt{t}, t)$ and $\sigma_{-}(t):=(-\sqrt{t},-\sqrt{t}, t)$ then

we get from [8], Corollary 4 , that

$$
\begin{aligned}
T_{\sigma_{+}, 1 / 2} V & =\left\{(x, y, z) \in \mathbb{C}^{3}: \frac{1}{2}\left(y\left(x^{2}-y^{2}\right)-4 y^{2}+(x+y)^{2}\right)=0\right\} \\
T_{\sigma_{+}, d} V & =\left\{(x, y, z) \in \mathbb{C}^{3}:-2 y^{2}+(x+y)^{2} / 2=0\right\}, \frac{1}{4}<d<\frac{1}{2}, \\
T_{\sigma_{+}, 1 / 4} V & =\left\{(x, y, z) \in \mathbb{C}^{3}: \frac{1}{2}(x+y)^{2}-2 y^{2}+1=0\right\} \\
T_{\sigma_{-}, 1 / 2} V & =\left\{(x, y, z) \in \mathbb{C}^{3}: \frac{1}{2}\left(y\left(x^{2}-y^{2}\right)+4 y^{2}-(x+y)^{2}\right)=0\right\} \\
T_{\sigma_{-}, d} V & =\left\{(x, y, z) \in \mathbb{C}^{3}: 2 y^{2}-(x+y)^{2} / 2=0\right\}, \frac{1}{4}<d<\frac{1}{2} \\
T_{\sigma_{-}, 1 / 4} V & =\left\{(x, y, z) \in \mathbb{C}^{3}: 2 y^{2}-(x+y)^{2} / 2+1=0\right\} .
\end{aligned}
$$

From the preceding considerations, (5.1), and the construction in 4.2 we now get

$$
\mathcal{K}_{2}=\left\{\left(\sigma_{+}, \frac{1}{2}, \frac{1}{4}\right),\left(\sigma_{-}, \frac{1}{2}, \frac{1}{4}\right)\right\}
$$

As (5.1) shows, $T_{\sigma_{ \pm}, 1 / 4} V$ is a manifold. Hence we have

$$
\mathcal{K}=\mathcal{K}_{1} \cup \mathcal{K}_{2}
$$

As $T_{\gamma_{ \pm}, 1 / 2} V$ and $T_{\sigma_{ \pm}, 1 / 4} V$ do not contain real terminating singularities, we have to show that $V$ is $\left(\gamma_{ \pm}, 1 / 2\right)$-hyperbolic at each $\xi \in\left(T_{\gamma_{ \pm}, 1 / 2} V\right)_{\text {reg }} \cap \mathbb{R}^{3}$ and that $V$ is $\left(\sigma_{ \pm}, \frac{1}{4}\right)$-hyperbolic at each $\xi \in\left(T_{\sigma_{ \pm}, 1 / 4} V\right)_{\text {reg }} \cap \mathbb{R}^{3}$. It is easy to check that these statements follow from Lemma 5.3 and the above equations defining these limit varieties. To complete the proof it therefore suffices to show that $V$ is $\left(\gamma_{ \pm}, 1, \frac{1}{2}\right)$ hyperbolic at $0 \in T_{\gamma_{ \pm}, 1} V$ and that $V$ is $\left(\sigma_{ \pm}, \frac{1}{2}, \frac{1}{4}\right)$-hyperbolic at $0 \in T_{\sigma_{ \pm}, 1 / 2} V$.

To prove the first assertion for $\gamma_{+}$, fix $z \in \mathbb{R}, z>1$, and $x \in \mathbb{R}$. We assume first that $x>2 \sqrt{z}$. Then it is easy to check that

$$
P(x,-2 x, z)>0, P(x, 0, z)<0, P(x, x, z)>0, P(x, 2 x, z)<0 .
$$

Hence $P$ has three real zeros $y_{j}(x, z), 1 \leq j \leq 3$, satisfying

$$
-2 x<y_{1}(x, z)<0<y_{2}(x, z)<x<y_{3}(x, z)<2 x .
$$


If $x<-2 \sqrt{z}$ then one can check that

$$
P(x, 2 x, z)>0, P(x, x / 2, z)<0, P(x, 0, z)>0, P(x,-2 x, z)<0 .
$$

As in the case $x>2 \sqrt{z}$, this implies that $P(x, \cdot, z)$ has three real zeros in the interval $] 2 x,-2 x\left[\right.$. Now define $\pi: \mathbb{C}^{3} \rightarrow \mathbb{C}^{3}, \pi(x, y, z):=(x, 0, z)$, and

$$
\Gamma:=\left\{(x, y, z) \in \mathbb{C}^{3}:|z|>1,\left|1-\frac{z}{|z|}\right|<\frac{1}{4},|x|<2|z|,|y|<2|z|\right\} .
$$

Then the considerations above show that $V$ is $\left(\gamma_{+}, 1,1 / 2\right)$-hyperbolic at $0 \in$ $T_{\gamma_{+}, 1} V$ in $\Gamma$ with respect to $\pi$.

The same arguments show that $V$ is $\left(\gamma_{-}, 1,1 / 2\right)$-hyperbolic at $0 \in T_{\gamma_{-}, 1} V$. In fact, a closer inspection shows that $V$ is even $\left(\gamma_{-}, 1\right)$-hyperbolic at $0 \in T_{\gamma_{-}, 1} V$ with respect to the projection $\pi$ which is given by $\operatorname{ker} \pi=\{(-\lambda, \lambda / 2,0): \lambda \in \mathbb{C}\}$ and $\operatorname{im} \pi=\left\{(\lambda, 2 \lambda, \zeta):(\lambda, \zeta) \in \mathbb{C}^{2}\right\}$.

Concerning the curves $\sigma_{ \pm}$we show more, namely, that $V$ is $\left(\sigma_{ \pm}, \frac{1}{2}\right)$-hyperbolic at $0 \in T_{\sigma_{ \pm}, 1 / 2} V$. We do this only for $\sigma_{+}$since the arguments are essentially the same for $\sigma_{-}$. To prove our claim, note first that $T_{0}\left(T_{\sigma_{+}, 1 / 2} V\right)=\left\{(x, y, z) \in \mathbb{C}^{3}\right.$ : $\left.(x+y)^{2} / 2-2 y^{2}=0\right\}$.

Hence the projection $\pi:(x, y, z) \mapsto(x, 0, z)$ is noncharacteristic at $0 \in$ $T_{\sigma_{+}, 1 / 2} V$. Then fix $z \in \mathbb{R}, z>1$, and $x \in \mathbb{R}$ satisfying $|x| \leq \sqrt{z} / 3$ and define

$$
\begin{aligned}
Q(x, y, z): & =P(\sqrt{z}+x, \sqrt{z}+y, z) \\
& =\frac{1}{2}(\sqrt{z}+y)(x-y)(x+y+2 \sqrt{z})+z(1-x+y) .
\end{aligned}
$$

We claim that

$$
Q(x,-3 \sqrt{z} / 4, z)<0, Q(x, x, z)=z>0, Q(x, 3 \sqrt{z} / 4, z)<0 .
$$

To prove this claim, note first that $\lambda \mapsto Q(\lambda,-3 \sqrt{z} / 4, z)$ is a quadratic polynomial in $\lambda$ with leading coefficient $\sqrt{z} / 8$, which is negative at $\lambda= \pm \sqrt{z} / 3$. Hence it must be negative for all $\lambda \in[-\sqrt{z} / 3, \sqrt{z} / 3]$, which implies the first inequality in (5.2) for $|x| \leq \sqrt{z} / 3$. The last inequality in (5.2) follows by similar arguments. Since $Q(x, x, z)=z$ is easy to check, our claim is proved. Obviously, it implies that for each $x$ satisfying $|x| \leq \sqrt{z} / 3$, the polynomial $y \mapsto Q(x, y, z)$ has two real zeros $y_{1,2}(x, z)$ satisfying $\left|y_{j}(x, z)\right|<3 \sqrt{z} / 4$. Since $Q(x, \cdot, z)$ is a cubic polynomial which satisfies $Q(x,-z, z)>0$ for $|x| \leq \sqrt{z} / 3$, its third zero $y_{3}(x, z)$ is also real and satisfies $\left|y_{3}(x, z)\right|>3 \sqrt{z} / 4$. From these facts it now follows easily that $V$ is $\left(\sigma_{+}, 1 / 2\right)$-hyperbolic at $0 \in T_{\sigma_{+}, 1 / 2} V$.

Example 5.6 Let $P_{5}, R, S, P$ and $Q \in \mathbb{C}[x, y, z]$ be defined as

$$
\begin{aligned}
P_{5}(x, y) & :=(x-y / 2)\left(x^{2}-y^{2}\right)\left(x^{2}-4 y^{2}\right) \\
R(x, y, z) & :=-y(y-3 x) z^{2}-z^{2}+1, \quad P:=P_{5}+R \\
S(x, y, z) & :=-y(y-3 x) z^{2}-z^{2}, \quad Q:=P_{5}+S .
\end{aligned}
$$

Then the following assertions hold: 
(a) $V(P)$ satisfies (SPL).

(b) $V(Q)$ does not satisfy (SPL).

Proof (a) To derive this from Theorem 4.3, note first that $\operatorname{grad} P$ does not vanish on $V(P)$. Hence we conclude as in the previous example that condition (1) of 4.3 (c) is satisfied. Then note that

$$
V_{h}=V\left(P_{5}\right)=\left\{(x, y, z) \in \mathbb{C}^{3}:(x-y / 2)\left(x^{2}-y^{2}\right)\left(x^{2}-4 y^{2}\right)=0\right\}
$$

is the union of five complex planes which have real generators. Hence $V_{h}$ satisfies (SPL) and consequently condition (2) of 4.3 (c) holds. To prove that also the conditions (3) and (4) of Theorem 4.3 are fulfilled, note first that

$$
V\left(P_{5}\right)_{\text {sing }} \cap S^{2}=\{(0,0,1),(0,0,-1)\}=: M_{0},
$$

and define $\gamma_{ \pm}(t):=(0,0, \pm t)$. Using [8], Corollary 4., it is easy to check that $V=V(P)$ has the following limit varieties:

$$
\begin{aligned}
& T_{\gamma_{ \pm}, d} V=\left\{(x, y, z) \in \mathbb{C}^{3}:(x-y / 2)\left(x^{2}-y^{2}\right)\left(x^{2}-4 y^{2}\right)=0\right\}, \quad \frac{2}{3}<d \leq 1 \\
& T_{\gamma_{ \pm}, \frac{2}{3}} V=\left\{(x, y, z) \in \mathbb{C}^{3}:(x-y / 2)\left(x^{2}-y^{2}\right)\left(x^{2}-4 y^{2}\right)-y(y-3 x)=0\right\} \\
& T_{\gamma_{ \pm}, d} V=\left\{(x, y, z) \in \mathbb{C}^{3}: y(y-3 x)=0\right\}, \quad 0<d<\frac{2}{3} \\
& T_{\gamma_{ \pm}, 0} V=\left\{(x, y, z) \in \mathbb{C}^{3}: y(y-3 x)+1=0\right\} \\
& T_{\gamma_{ \pm}, d} V=\emptyset, \quad-\infty<d<0 .
\end{aligned}
$$

Since $V(P)$ has no singular points, it follows as in Example 5.5 that the points in $M_{0}$ are not terminating. At each regular point $\xi \in V_{h} \cap S^{n-1}$ we can use Lemma 5.3 to see that $V$ is $\left(\gamma_{\xi}, 1\right)$-hyperbolic at 0 for $\gamma_{\xi}(t):=t \xi$. Hence condition (3) of 4.3 (c) holds.

Next note that by the definition of the set $\mathcal{K}_{1}$ in 4.2 , we have

$$
\mathcal{K}_{1}=\left\{\left(\gamma_{+}, 1, \frac{2}{3}\right),\left(\gamma_{-}, 1, \frac{2}{3}\right)\right\}
$$

From (5.3) we get

$$
\left(T_{\gamma_{ \pm}, 2 / 3} V\right)_{\text {sing }} \cap \mathbb{R}^{3}=\{(0,0, z): z \in \mathbb{R}\} .
$$

Since all points of $V(P)$ are regular, the singular point $(0,0,0) \in T_{\gamma_{ \pm}, 2 / 3} V$ is not terminating for $\gamma_{ \pm}$and $2 / 3$. Thus we have $\mathcal{K}_{1, c}=\mathcal{K}$ and it follows from (5.3) and the construction in 4.2 that

$$
\mathcal{K}_{2}=\left\{\left(\gamma_{+}, \frac{2}{3}, 0\right),\left(\gamma_{-}, \frac{2}{3}, 0\right)\right\} .
$$

Since all points of $T_{\gamma_{ \pm}, 0} V$ are regular, we have $\mathcal{K}_{2, c}=\emptyset$. Thus we showed that $\mathcal{K}=\mathcal{K}_{1} \cup \mathcal{K}_{2}$.

Next note that $T_{\gamma_{ \pm}, 2 / 3} V$ does not contain any real singular points which are terminating and that $T_{\gamma_{ \pm}, 0} V$ does not contain any singular points at all. Furthermore, 
by Lemma 5.3, $V$ is $\left(\gamma_{ \pm}, 2 / 3\right)$-hyperbolic at each $\xi \in\left(T_{\gamma_{ \pm}, 2 / 3} V\right)_{\text {reg }} \cap \mathbb{R}^{3}$ and $\left(\gamma_{ \pm}, 0\right)$-hyperbolic at each $\xi \in\left(T_{\gamma_{ \pm}, 0} V\right)_{\text {reg }} \cap \mathbb{R}^{3}$. Hence it remains to show that $V$ is $\left(\gamma_{ \pm}, 1,2 / 3\right)$-hyperbolic at $0 \in T_{\gamma_{ \pm}, 1} V$ and that $V$ is $\left(\gamma_{ \pm}, 2 / 3,0\right)$-hyperbolic at $0 \in T_{\gamma_{ \pm}, 2 / 3} V$.

To show that $V$ is $\left(\gamma_{+}, 1,2 / 3\right)$-hyperbolic at $0 \in T_{\gamma_{+}, 1} V$, note first that the projection $\pi(x, y, z):=(x, 0, z)$ is noncharacteristic for $T_{\gamma_{+}, 1} V$ at 0 or equivalently for $V_{h}$ at $(0,0,1)$. Then some computation shows that for $z \geq 27$ and $x \geq 2 z^{2 / 3}$ we have

$$
\begin{aligned}
P(x, 3 x, z) & <0, P(x, x, z)>0, P(x, 3 x / 4, z)<0, \\
P(x, 0, z) & >0, P(x,-3 x / 4, z)<0, \text { and } P(x,-2 x, z)>0,
\end{aligned}
$$

while for $x<-z^{2 / 3}$ we have

$$
\begin{gathered}
P(x,-x, z)<0, P(x,-3 x / 4, z)>0, P(x, 0, z)<0, \\
P(x, 3 x / 4, z)>0, P(x, 4 x / 3, z)<0, \text { and } P(x, 3 x, z)>0 .
\end{gathered}
$$

From this it follows as in the previous example that $V$ is $\left(\gamma_{+}, 1,2 / 3\right)$-hyperbolic at $0 \in T_{\gamma_{+}, 1} V$. Since $P$ is an even function of $z$, the same arguments apply for $\gamma_{-}$.

To derive the $(\gamma \pm, 2 / 3,0)$-hyperbolicity of $V$, note first that the projection $\pi$ from above is also noncharacteristic for $T_{\gamma_{ \pm}, 2 / 3} V$. To determine the real zeros of $V$ in the conoid $\Gamma\left(\gamma_{+}, \frac{2}{3}, G, r\right)$ for a suitable zero neighborhood $G$, we consider the equation

$$
\begin{aligned}
0 & =P\left(t^{2 / 3} x, t^{2 / 3} y, t\right) / t^{10 / 3} \\
& =(x-y / 2)\left(x^{2}-y^{2}\right)\left(x^{2}-4 y^{2}\right)-y(y-3 x)-t^{-4 / 3}+t^{-10 / 3} .
\end{aligned}
$$

Using the theorem of Rouché it is easy to check that for $t \geq 1$ and $t^{-2 / 3} \leq|x| \leq \frac{1}{20}$ and $x$ real, this equation has exactly two solutions $y_{1}(x), y_{2}(x) \in B(0,1 / 5)$. Since the roots of the equation $y(y-3 x)=0$ are real and distinct in this range of $x$, a standard application of the real and the complex implicit function theorem shows that $y_{1}(x)$ and $y_{2}(x)$ must be real for real $x$. Rescaling these assertions, we get that $V$ is $\left(\gamma_{+}, 2 / 3,0\right)$-hyperbolic at 0 . For $\gamma_{-}$the same arguments apply.

(b) To show that $V(Q)$ does not satisfy $\mathrm{PL}_{\mathrm{loc}}(0)$, note that the tangent cone to $V(Q)$ at zero is given by

$$
T_{0} V(Q)=\left\{(x, y, z) \in \mathbb{C}^{3}: z=0\right\} .
$$

Hence the projection $\pi(x, y, z):=(x, y, 0)$ is noncharacteristic for $T_{0} V(Q)$ at zero. Next define $\gamma(t):=(-t, 0,0)$ for $0 \leq t \leq 1$ and note that

$$
\pi^{-1}(\gamma(t)) \cap V(Q) \supset\left\{\left(-t, 0, i t^{5 / 2}\right),\left(-t, 0,-i t^{5 / 2}\right)\right\}, 0<t<1 .
$$

Since these points belong to $\Gamma(\gamma, 1, B(0, \varepsilon), r)$ for each $\varepsilon>0$ and $0<r \leq r(\varepsilon)$, we proved that $V(Q)$ is not $(\gamma, 1)$-hyperbolic at $0 \in T_{\gamma, 1} V(Q)$, in the notation of [5]. Hence it follows from [5], Proposition 3.12, that $V(Q)$ does not satisfy $\mathrm{PL}_{\mathrm{loc}}(0)$. Since the latter condition is necessary for $V(Q)$ to satisfy (SPL) by Proposition 3.4, we proved (b). 
Next we indicate how the results of this paper can be used to derive the main theorem of [3]. For that purpose, recall that for $P \in \mathbb{C}\left[z_{1}, \ldots, z_{n}\right]$ and $\theta \in \mathbb{C}^{n}$ the localization $P_{\theta}$ of $P$ at $\theta$ is defined as the lowest degree homogeneous term in the Taylor expansion of $P$ at $\theta$.

Lemma 5.7 Let $P_{m} \in \mathbb{C}\left[z_{1}, \ldots, z_{n}\right]$ be homogeneous of degree $m \geq 2$ and define $V_{+}:=\left\{z \in \mathbb{C}^{n}: P_{m}(z)=1\right\}$. If $V_{+}$satisfies (SPL) then for each $\theta \in V\left(P_{m}\right) \cap S^{n-1}$ the variety

$$
W_{\theta,+}:=\left\{z \in \mathbb{C}^{n}:\left(P_{m}\right)_{\theta}(z)=1\right\}
$$

satisfies (SPL).

Proof Fix $\theta \in V\left(P_{m}\right) \cap S^{n-1}$. After a real linear change of variables we may assume that $\theta=(0, \ldots, 0,1)$. Then we expand

$$
P_{m}\left(z^{\prime}, z_{n}\right)=\sum_{j=v}^{m} Q_{j}\left(z^{\prime}\right) z_{n}^{m-j}
$$

where $Q_{j} \in \mathbb{C}\left[z_{1}, \ldots, z_{n-1}\right]$ is either homogeneous of degree $j$ or identically zero and where $Q_{v} \not \equiv 0$. By [3], Lemma 3.9, we have $\left(P_{m}\right)_{\theta}\left(z^{\prime}, z_{n}\right)=Q_{v}\left(z^{\prime}\right)$. Next define $\gamma(t):=(0, \ldots, 0, t)$ and consider for $P:=P_{m}-1$ the expansion

$$
\begin{aligned}
P(\gamma(t)+z) & =\sum_{j=v}^{m} Q_{j}\left(z^{\prime}\right)\left(t+z_{n}\right)^{m-j}-1 \\
& =\sum_{j=v}^{m} Q_{j}\left(z^{\prime}\right) \sum_{k=0}^{m-j}\left(\begin{array}{c}
m-j \\
k
\end{array}\right) t^{k} z_{n}^{m-j-k}-1 .
\end{aligned}
$$

From the Newton diagram of this expansion and [8], Corollary 4, it follows that we have

$$
\begin{aligned}
T_{\gamma, 1} V_{+} & =\left\{\left(z^{\prime}, z_{n}\right) \in \mathbb{C}^{n}: P_{m}\left(z^{\prime}, z_{n+1}\right)=0\right\}, \\
T_{\gamma, d} V_{+} & =\left\{\left(z^{\prime}, z_{n}\right) \in \mathbb{C}^{n}: Q_{v}\left(z^{\prime}\right)=0\right\}, 1-\frac{m}{v}<d<1, \\
T_{\gamma, 1-\frac{m}{v}} V_{+} & =\left\{\left(z^{\prime}, z_{n}\right) \in \mathbb{C}^{n}: Q_{\nu}\left(z^{\prime}\right)-1=0\right\} .
\end{aligned}
$$

By Proposition 3.5, all these varieties satisfy (SPL). Since $\left(P_{m}\right)_{\theta}=Q_{\nu}$, the proof of the lemma is complete.

Lemma 5.8 Let $Q_{m} \in \mathbb{C}\left[z_{1}, \ldots, z_{n}\right]$ be homogeneous of degree $m$ and define $W_{ \pm}:=\left\{z \in \mathbb{C}^{n}: Q_{m}(z)= \pm 1\right\}$. If both varieties $W_{+}$and $W_{-}$satisfy (SPL) then $Q_{m}$ is square-free.

Proof To argue by contradiction, we assume that $Q_{m}=R^{k} S$ for suitable $R, S \in$ $\mathbb{C}\left[z_{1}, \ldots, z_{n}\right], R$ not constant and $k \geq 2$. By Proposition 3.5, the present hypotheses imply that $V\left(Q_{m}\right)$ and consequently $V(R)$ and $V(S)$ satisfy (SPL). Therefore, we may assume by Lemma 3.17 (b) that $R$ and $S$ have real coefficients. Moreover, we can choose a regular point $\xi \in V\left(Q_{m}\right) \cap S^{n-1}$ which is in $V(R)$. Then we define 
the real simple curve $\gamma$ by $\gamma(t):=\xi t$. By Proposition 3.9, the present hypothesis implies that $W_{+}$and $W_{-}$are both $(\gamma, 1)$-hyperbolic at $0 \in T_{\gamma, 1} W_{ \pm}=V\left(Q_{m}\right)-\xi$ for each projection $\pi: \mathbb{C}^{n} \rightarrow \mathbb{C}^{n}$ which is noncharacteristic for $T_{\gamma, 1} W_{ \pm}$at the origin. Fix such a projection $\pi$ which is even noncharacteristic for $V\left(Q_{m}\right)$ at infinity. Choosing appropriate coordinates, we may assume $\xi=(1,0, \ldots, 0)$, $\pi\left(z^{\prime}, z_{n}\right)=\left(z^{\prime}, 0\right)$, and $Q_{m}(0, \ldots, 0,1) \neq 0$. Then $p \in \mathbb{C}[t, z]$, defined by

$$
p(t, z):=Q_{m}(t, 0, \ldots, 0, z),
$$

is homogeneous of degree $m$ and vanishes on the line $\mathbb{C} \backslash\{0\}$ of order $q \geq k \geq 2$. Hence there exist $c, a_{1}, \ldots, a_{m-q} \in \mathbb{C} \backslash\{0\}$ such that

$$
p(t, z)=c z^{q} \prod_{j=1}^{m-q}\left(z-a_{j} t\right) .
$$

Consequently, for each $\varepsilon>0$ there exists $t_{0}>1$ such that for each $t \geq t_{0}$ the two equations

$$
p(t, z) \pm 1=0
$$

have $q$ solutions $z_{j}^{ \pm}(t) \in B(0, \varepsilon t), 1 \leq j \leq q$. They admit a Puiseux series expansion of the form

$$
\gamma(t)=\sum_{\nu=-\infty}^{\kappa-1} b_{\nu} t^{\nu / \kappa}
$$

for some $\kappa \in \mathbb{N}$. A standard computation shows that the first nonvanishing term in such an expansion is given by

$$
\left(\mp c\left(\prod_{j=1}^{m-q}\left(-a_{j}\right)\right) t^{m-q}\right)^{-1 / q} .
$$

Because of $q \geq 2$ there is a choice of + or - such that the coefficient of this term is in $\mathbb{C} \backslash \mathbb{R}$. Hence for each $t \geq t_{0}$ not all solutions in $B(0, \varepsilon t)$ of both equations in (5.5) are real. Since this contradicts the $(\gamma, 1)$-hyperbolicity of $W_{ \pm}$at $0 \in T_{\gamma, 1} W_{ \pm}$, the proof is complete.

Proposition 5.9 Let $P_{m} \in \mathbb{C}\left[z_{1}, \ldots, z_{n}\right]$ be homogeneous of degree $m \geq 2$ and define $V_{ \pm}:=\left\{z \in \mathbb{C}^{n}: P_{m}(z)= \pm 1\right\}$.

(a) If $V_{+}$and $V_{-}$both satisfy (SPL), then $V\left(P_{m}\right)$ satisfies (SPL), $P_{m}$ has real coefficients, and for each $\theta \in V\left(P_{m}\right) \cap S^{n-1}$ the localization of $P_{m}$ at $\theta$ is square-free.

(b) For $n=3$ the necessary conditions in (a) imply that $V_{+}$and $V_{-}$satisfy (SPL).

Proof (a) The conditions stated in (a) hold by Proposition 3.5, Lemma 3.17 (b), Lemma 5.7, and Lemma 5.8 since $V_{-}=V_{+}\left(Q_{m}\right)$ for $Q_{m}:=-P_{m}$ and since $\left(Q_{m}\right)_{\theta}=-\left(P_{m}\right)_{\theta}$.

(b) To derive (b) from Theorem 4.3 we show that the conditions (1)-(4) in part

(c) of this theorem are fulfilled. 
(1) By Euler's rule we have $\left\langle x, \operatorname{grad} P_{m}(x)\right\rangle=m P_{m}(x)$ for all $x \in \mathbb{C}^{n}$. Hence $\operatorname{grad} P_{m}(x)=0$ implies $P_{m}(x)=0$. Consequently, each point of $V_{ \pm}$is a regular point. Since $P_{m}$ has real coefficents it follows that $V_{ \pm}$satisfies $\mathrm{PL}_{\text {loc }}(\xi)$ at each $\xi \in V_{ \pm} \cap \mathbb{R}^{3}$.

(2) This holds by hypothesis.

(3) As we remarked in (1), there is no singular curve in $V_{ \pm}$. Hence no point in $\left(V_{h}\right)_{\text {sing }} \cap S^{2}$ can be terminating by the argument that we used in Example 5.6. At each point in $\left(V_{h}\right)_{\text {reg }} \cap S^{2}$, the gradient of $P_{m}$ does not vanish because of the present hypotheses. Hence (3) follows from Lemma 5.3.

(4) Let $M_{0}:=\left\{\xi \in V\left(P_{m}\right)_{\text {sing }} \cap S^{2}\right\}$. For $\xi \in M_{0}$ and $\gamma(t)=\xi t$, we have by (5.4)

$$
T_{\gamma, 1-\frac{m}{v}} V_{ \pm}=\left\{z \in \mathbb{C}^{n}:\left(P_{m}\right)_{\xi}(z)= \pm 1\right\} \text { where } v=\operatorname{deg}\left(P_{m}\right)_{\xi}
$$

and all points of this set are regular. Hence $\mathcal{K}=\mathcal{K}_{1}=\left\{\left(\gamma_{\xi}, 1, \Delta\left(\gamma_{\xi}, 1,0\right)\right): \xi \in\right.$ $\left.M_{0}\right\}$. By Lemma 5.3 the $\left(\gamma_{\xi}, \Delta\left(\gamma_{\xi}, 1,0\right)\right)$-hyperbolicity condition in (4) is fulfilled. The fact that $V$ is $\left(\gamma_{\xi}, 1, \Delta\left(\gamma_{\xi}, 1,0\right)\right)$-hyperbolic was proved in [3], Lemma 5.6.

Combining Proposition 5.9 with the results from Meise and Taylor [14] we obtain now the main result of [3] namely Theorem 1.1.

Theorem 5.10 Let $P_{m} \in \mathbb{C}\left[z_{1}, z_{2}, z_{3}\right]$ be homogeneous of degree $m \geq 2$ and define $P \in \mathbb{C}\left[z_{1}, \ldots, z_{4}\right]$ by $P(z):=P_{m}\left(z_{1}, z_{2}, z_{3}\right)-z_{4}$. The following assertions are equivalent:

(1) $V(P)$ has (SPL),

(2) $P(D): C^{\infty}\left(\mathbb{R}^{4}\right) \rightarrow C^{\infty}\left(\mathbb{R}^{4}\right)$ admits a continuous linear right inverse,

(3) $V_{+}\left(P_{m}\right)$ and $V_{-}\left(P_{m}\right)$ satisfy (SPL),

(4) $V\left(P_{m}\right)$ has $(\mathrm{SPL}), P_{m}$ has real coefficients, and $\left(P_{m}\right)_{\theta}$ is square-free for each $\theta \in V\left(P_{m}\right) \cap \mathbb{R}^{3}$ with $|\theta|=1$.

Proof $(1) \Rightarrow(2)$ : Since the condition (SPL) for $V(P)$ obviously implies that $V(P)$ satisfies $\operatorname{PL}\left(\mathbb{R}^{4}, \log \right)$ (see [3], Definition 2.4) and since PL( $\left.\mathbb{R}^{4}, \log \right)$ is equivalent to (2), this implication holds.

$(2) \Rightarrow(3) \Rightarrow(1)$ : As we already remarked, $V(P)$ satisfies $\operatorname{PL}\left(\mathbb{R}^{4}, \log \right)$. By [16], Theorem 4.1 , this implies that also $V\left(P_{m}\right) \times \mathbb{C}$ satisfies $\operatorname{PL}\left(\mathbb{R}^{4}, \log \right)$. Since $V\left(P_{m}\right)$ is homogeneous, $V\left(P_{m}\right)$ even satisfies (SPL). By Lemma $3.17(\mathrm{~b})$, there exists $\lambda \in \mathbb{C},|\lambda|=1$ such that $\lambda P_{m} \in \mathbb{R}\left[z_{1}, z_{2}, z_{3}\right]$. Since $V(\lambda P)=V(P)$ satisfies $\operatorname{PL}\left(\mathbb{R}^{4}, \log \right)$, it follows from Meise and Taylor [14], Lemma 2.1, that $\lambda= \pm 1$. Thus, $P_{m}$ has real coefficients and the desired implications hold by Meise and Taylor [14], Theorem 3.4.

(3) $\Rightarrow(4) \Rightarrow(3)$ : These implications hold by Proposition 5.9.

\section{References}

1. Bochnak, J., Coste, M., Roy, M.-F.: Géometrie algébrique réelle, Ergebnisse Math. Grenzgebiete, 3. Folge, Band 12, Springer, Berlin 1987

2. Braun, R. W.: Hörmander's Phragmén-Lindelöf principle and irreducible singularities of codimension 1. Boll. Un. Mat. Ital. 6-A(7), 339-348 (1992) 
3. Braun, R.W., Meise, R., Taylor, B.A.: Algebraic varieties on which the classical PhragménLindelöf estimates hold for plurisubharmonic function. Math. Z. 232, 103-135 (1999)

4. Braun, R.W., Meise, R., Taylor, B.A.: A radial Phragmén-Lindelöf estimate for plurisubharmonic functions on algebraic varieties. Ann. Polon. Math. 72, 159-179 (1999)

5. Braun, R.W., Meise, R., Taylor, B.A.: The geometry of analytic varieties satisfying the local Phragmén-Lindelöf condition and a geometric characterization of the partial differential operators that are surjective on $\mathcal{A}\left(\mathbb{R}^{4}\right)$. Trans. Amer. Math. Soc. 356, 1315-1383 (2004)

6. Braun, R.W., Meise, R., Taylor, B.A.: Optimal Gevrey classes for the existence of solution operators for linear partial differential operators in three variables. J. Math. Anal. Appl. 297, 852-868 (2004)

7. Braun, R.W., Meise, R., Taylor, B.A.: Characterization of the linear partial differential equations that admit solution operators on Gevrey classes. J. reine angew. Math.

8. Braun, R.W., Meise, R., Taylor, B.A.: Higher order approximation at infinity to algebraic varieties. Note di Mat.

9. Braun, R.W., Meise, R., Taylor, B.A.: Higher order tangents to analytic varieties along curves II. Canad. J. Math., to appear

10. Chirka, E.M.: Complex Analytic sets, Kluwer, Dordrecht, 1989

11. Heinrich, T.: A new necessary condition for analytic varieties satisfying the local PhragménLindelöf condition. Ann. Pol. Math. 85, 283-290 (2005)

12. Hörmander, L.: On the existence of real analytic solutions of partial differential equations with constant coefficients. Invent. Math. 21, 151-183 (1973)

13. Hörmander, L.: The Analysis of Linear Partial Differential Operators II. Springer, Berlin, 1983

14. Meise, R., Taylor, B.A.: Phragmén-Lindelöf conditions for graph varieties. Result. Math. 36, 121-148 (1999)

15. Meise, R., Taylor, B.A., Vogt, D.: Extremal plurisubharmonic functions of linear growth on algebraic varieties. Math. Z. 219, 515-537 (1995)

16. Meise, R., Taylor, B.A., Vogt, D.: Phragmén-Lindelöf principles on algebraic varieties. J. Amer. Math. Soc. 11, 1-39 (1998)

17. Whitney, H.: Complex analytic varietes. Addison-Wesley Pub. Co., 1972 